\title{
CD56 bright NK cells exhibit potent antitumor responses following IL-15 priming
}

\author{
Julia A. Wagner, ${ }^{1}$ Maximillian Rosario, ${ }^{1}$ Rizwan Romee, ${ }^{1}$ Melissa M. Berrien-Elliott, ${ }^{1}$ Stephanie E. Schneider, ${ }^{1}$ Jeffrey W. Leong, ${ }^{1}$ \\ Ryan P. Sullivan,, Brea A. Jewell, ${ }^{1}$ Michelle Becker-Hapak, ${ }^{1}$ Timothy Schappe, ${ }^{1}$ Sara Abdel-Latif, ${ }^{1}$ Aaron R. Ireland, ${ }^{1}$ \\ Devika Jaishankar, ${ }^{1}$ Justin A. King, ${ }^{1}$ Ravi Vij, ${ }^{1}$ Dennis Clement,, ${ }^{2,3}$ Jodie Goodridge, ${ }^{2}$ Karl-Johan Malmberg, ${ }^{2,3,4}$ \\ Hing C. Wong, ${ }^{5}$ and Todd A. Fehniger ${ }^{1}$ \\ 'Department of Medicine, Division of Oncology, Washington University School of Medicine, St. Louis, Missouri, USA. ²Department of Cancer Immunology, Institute for Cancer Research, \\ Oslo University Hospital, Radiumhospitalet, Oslo, Norway. ${ }^{3}$ The KG Jebsen Centre for Cancer Immunotherapy, Institute of Clinical Medicine, University of Oslo, 0slo, Norway. \\ ${ }^{4}$ Centre for Infectious Medicine, Department of Medicine Huddinge, Karolinska Institutet, Stockholm, Sweden. ${ }^{5}$ Altor BioScience, Miramar, Florida, USA.
}

\begin{abstract}
NK cells, lymphocytes of the innate immune system, are important for defense against infectious pathogens and cancer. Classically, the CD56 ${ }^{\text {dim }}$ NK cell subset is thought to mediate antitumor responses, whereas the CD56 bright subset is involved in immunomodulation. Here, we challenge this paradigm by demonstrating that brief priming with IL-15 markedly enhanced the antitumor response of CD56 ${ }^{\text {bright }}$ NK cells. Priming improved multiple CD56 bright cell functions: degranulation, cytotoxicity, and cytokine production. Primed CD56 bright cells from leukemia patients demonstrated enhanced responses to autologous blasts in vitro, and primed CD56 bright cells controlled leukemia cells in vivo in a murine xenograft model. Primed CD56 bright cells from multiple myeloma (MM) patients displayed superior responses to autologous myeloma targets, and furthermore, CD56 bright NK cells from MM patients primed with the IL-15 receptor agonist ALT-803 in vivo displayed enhanced ex vivo functional responses to MM targets. Effector mechanisms contributing to IL-15-based priming included improved cytotoxic protein expression, target cell conjugation, and LFA-1-, CD2-, and NKC2D-dependent activation of NK cells. Finally, IL-15 robustly stimulated the PI3K/Akt/mTOR and MEK/ERK pathways in CD56 bright compared with CD56 dim NK cells, and blockade of these pathways attenuated antitumor responses. These findings identify CD56 bright $N K$ cells as potent antitumor effectors that warrant further investigation as a cancer immunotherapy.
\end{abstract}

\section{Introduction}

NK cells are innate lymphoid cells important for host defense against infectious pathogens and cancer (1). The NK cell response to a potential target relies on the integration of activating and inhibitory receptor signals induced by interaction with the target cell $(2,3)$, memory of prior exposures $(4)$, the cytokine milieu (5), and education during development (6). Human NK cells are identified via surface expression of CD56 and lack of CD3, and can be categorized into 2 developmentally related, but functionally distinct, subsets based on relative CD56 expression: CD56 ${ }^{\text {bright }}$ and $\operatorname{CD}^{\mathrm{dim}}(1,7,8)$. The CD56 $6^{\mathrm{dim}}$ subset comprises $80 \%-95 \%$ of peripheral blood NK cells. CD56 ${ }^{\mathrm{dim}}$ NK cells represent the final stage of NK cell maturation (stage V), and as a result express maturity-related inhibitory receptors (killer cell immunoglobulinlike receptors [KIRs]), cytotoxic effector proteins (including perforin and granzyme B) at rest, and high surface levels of CD16

Authorship note: J.A. Wagner and M. Rosario contributed equally to this work. Conflict of interest: H.C. Wong is employed by and has equity interest in Altor BioScience. K.J. Malmberg serves on the Scientific Advisory Board of Fate Therapeutics. All relationships have been reviewed and managed by Oslo University Hospital and Karolinska Institute in accordance with its conflict of interest polices.

Submitted: August 31, 2016; Accepted: August 15, 2017.

Reference information: J Clin Invest. 2017;127(11):4042-4058.

https://doi.org/10.1172/JCI90387.
(Fc $\gamma$ RIIIa), permitting responses to antibody-opsonized targets. The less mature (stage IV) CD56 $6^{\text {bright }}$ NK cells are a minor subset in the peripheral blood, but predominate in secondary lymphoid tissues. Indeed, CD56 $6^{\text {bright }} \mathrm{NK}$ cells are estimated to outnumber CD56 ${ }^{\text {dim }}$ NK cells overall (9-11). CD56 $6^{\text {bright }}$ NK cells express lower levels of cytotoxic effector proteins at rest, partially express CD16, rely on CD94/NKG2A receptors rather than KIRs for selftolerance, and possess distinct chemokine and homing receptor repertoires compared with the CD56 $6^{\mathrm{dim}}$ subset. CD56 $6^{\text {bright }} \mathrm{NK}$ cells are potent cytokine producers (IFN- $\gamma$, TNF, GM-CSF) following combined cytokine receptor stimulation $(7,12,13)$ but, unlike CD56 ${ }^{\mathrm{dim}} \mathrm{NK}$ cells, display minimal antitumor responsiveness at rest. As a result of these properties, CD56 $6^{\text {bright }} \mathrm{NK}$ cells are traditionally considered ineffective antitumor responders that instead function primarily in immunomodulation.

Studies from a number of laboratories have established the antitumor potential of NK cells, chiefly in the setting of allogeneic hematopoietic cell transplantation (HCT) (14) or adoptive NK cell transfer $(15,16)$, inspiring substantial interest in NK cellbased immunotherapy $(17,18)$. In addition, reports that donor KIR haplotype can influence allogeneic HCT outcomes provide correlative evidence that NK cells mediate a graft-versusleukemia effect in vivo (19-21). However, these studies typically do not investigate which NK cell subset is responsible for mediating antitumor responses, and functional analyses have focused 
on $\mathrm{KIR}^{+} \mathrm{NK}$ cells, leaving the antitumor potential of $\mathrm{CD} 56^{\text {bright }}$ NK cells relatively unexplored.

IL-15 is a central cytokine in NK cell development, homeostasis, and function $(1,22)$. There are three IL-15 receptor subunits: IL-15R $\alpha, \beta$, and $\gamma$. The IL-15 receptor shares its $\beta$ subunit with IL-2, and its $\gamma$ subunit (common $\gamma$ chain) with IL-2, -4, -7, -9, and -21. The IL- $15 \mathrm{R} \beta / \gamma$ heterodimer is an intermediate- (nanomolar-) affinity IL-15 receptor constitutively expressed by both CD $56^{\text {bright }}$ and CD56 ${ }^{\mathrm{dim}} \mathrm{NK}$ cells that functions in ligand binding and signal transduction. IL-15R $\alpha$ binds IL-15 with high (picomolar) affinity. IL-15/IL-15R $\alpha$ can associate with IL-15R $\beta / \gamma$ either on the same cell (cis-presentation) or on a different cell (trans-presentation) to initiate signaling, although trans-presentation is thought to be the major IL-15R trigger in vivo $(22,23)$. Three primary pathways are activated downstream of the IL-15R: JAK1/3/STAT3/5, PI3K/Akt/ mTOR, and Ras/Raf/MEK/ERK $(23,24)$. Through these pathways, IL-15 has been shown to promote the survival and proliferation of CD56 $6^{\text {bright }}$ and CD56 $6^{\text {dim }} \mathrm{NK}$ cells, and to enhance the cytotoxicity of the CD56 $6^{\mathrm{dim}}$ subset (25). Prolonged exposure to IL-2, which also acts through the intermediate-affinity IL-2/15R $\beta \gamma$, has similarly been shown to increase NK cell cytotoxicity (25). However, IL-2 also promotes regulatory $\mathrm{T}$ cell responses, and may thereby limit antitumor immunity $(26,27)$. Thus, while IL-15 supports the survival and proliferation of CD56 $6^{\text {bight }} \mathrm{NK}$ cells (22), its impact on the antitumor responses of this human NK cell subset has not been well examined.

In a model organism, we and others have previously demonstrated that brief priming of murine NK cells with IL-15 optimizes functional responses to tumor or virus-infected targets, including cytokine production and killing $(28,29)$. Since in some ways the CD56 ${ }^{\text {bright }} \mathrm{NK}$ cell cytotoxic program resembles that of specific pathogen-free murine NK cells (28), we reasoned that IL-15 may globally enhance CD56 $6^{\text {bright }} \mathrm{NK}$ cell antitumor responses. Here, we demonstrate that brief priming of CD56 $6^{\text {bright }} \mathrm{NK}$ cells with IL-15 results in markedly increased degranulation, cytotoxicity, and effector cytokine production in response to tumor targets in vitro and in vivo. This priming effect was seen for normal-donor CD $56^{\text {bright }} \mathrm{NK}$ cells, as well as CD56 $6^{\text {bright }} \mathrm{NK}$ cells of patients with hematologic malignancies triggered by autologous tumor cells. Several mechanisms were identified that underlie the enhanced antitumor responsiveness of these polyfunctional IL-15-primed CD56 ${ }^{\text {bright }}$ NK cells. Moreover, we link preferential induction of $\mathrm{PI} 3 \mathrm{~K} / \mathrm{Akt} / \mathrm{mTOR}$ and MEK/ERK signaling by IL-15 in CD56 $6^{\text {bright }}$, compared with $\mathrm{CD} 56^{\mathrm{dim}}$, NK cells with enhanced function. Blockade of these pathways attenuated IL-15 priming, identifying a molecular mechanism responsible for this priming event. Our findings suggest that in clinical settings of increased IL-15, CD56 ${ }^{\text {bright }} \mathrm{NK}$ cells may exhibit potent multifunctional antitumor responses, and that this NK cell subset could be harnessed for cancer immunotherapy.

\section{Results}

IL-15 primes human CD56 bright $N K$ cells for enhanced antitumor responses. Purified NK cells from normal donors were cultured for 12-16 hours with (primed) or without (control) recombinant human (rh) IL-15 at $5 \mathrm{ng} / \mathrm{ml}$ (a concentration that induces signaling via the IL-2/15R $\beta \gamma$ ) (22). Cells were then washed and trig- gered with tumor targets for 6 hours, after which degranulation (CD107a surface expression) and cytokine production (intracellular IFN- $\gamma$ and TNF expression) were assessed (Figure 1A). IL-15 priming robustly enhanced degranulation and cytokine production by CD $56^{\text {bright }} \mathrm{NK}$ cells in response to K562 acute myeloid leukemia (AML) target cells (Figure 1B). Indeed, while IL-15 priming also modestly enhanced CD56 $6^{\mathrm{dim}} \mathrm{NK}$ cell antitumor responses, these were surpassed by those of IL-15-primed CD56 $6^{\text {bright }}$ NK cells (Figure 1B). Similar responses to K562 target cells were observed with flow-sorted, control or IL-15-primed CD56 $6^{\text {bright }}$ and CD56 ${ }^{\mathrm{dim}} \mathrm{NK}$ cells (Figure 1C), indicating that IL-15 priming of CD56 $6^{\text {bright }} \mathrm{NK}$ cell antitumor responses was not a result of competition for IL-15 or differential effector-to-target (E:T) ratios. IL-15 priming of CD56 bright NK cells could be observed after as little as 1 hour of stimulation (Supplemental Figure 1A; supplemental material available online with this article; https://doi.org/10.1172/JCI90387DS1), and with IL-15 concentrations as low as $1 \mathrm{ng} / \mathrm{ml}$ (Supplemental Figure 1B). In addition, a large proportion of IL-15-primed CD56 $6^{\text {bright }} \mathrm{NK}$ cells exhibited polyfunctional responses, with simultaneous degranulation and IFN- $\gamma$ and TNF production in response to K562 target cells (Supplemental Figure 2, A and B). Enhanced responses of IL-15primed CD56 $6^{\text {bright }} \mathrm{NK}$ cells were also confirmed against the $\mathrm{MHC}$ class I-expressing HL-60 AML cell line (Supplemental Figure 2C).

Since IL-15 is critical for NK cell homeostasis as well as function, we also established that CD56 $6^{\text {bright }} \mathrm{NK}$ cell responses immediately following purification were equivalent to those after overnight culture in media alone (control NK cells) from the same donor (data not shown). Additionally, after 16 hours there was no difference in NK cell viability between control and primed conditions. However, IL-15 priming selectively enhanced CD56 $6^{\text {bright }}$ NK cell survival in the setting of cytokine withdrawal (Supplemental Figure 3). Brief IL-15 priming was not sufficient to induce substantial proliferation of CD56 $6^{\text {bright }}$ or CD56 $6^{\mathrm{dim}} \mathrm{NK}$ cells (Supplemental Figure 4), suggesting that NK cell proliferation pathways require prolonged IL-15R signals to promote cell division in CD56 $6^{\text {bright }}$ and CD56 ${ }^{\mathrm{dim}} \mathrm{NK}$ cells.

IL-15 priming of CD56 ${ }^{\text {dim }} \mathrm{NK}$ cells is in part maturity-dependent. While CD56 $6^{\mathrm{dim}} \mathrm{NK}$ cells are considered more differentiated than the $\mathrm{CD} 56^{\text {bright }}$ subset, they still exist within a range of maturity states. NK cell maturity can be delineated by the presence or absence of specific surface markers such as KIR, NKG2A, CD57, CD11b, and CD27 $(30,31)$. We hypothesized that IL-15 priming of CD56 ${ }^{\mathrm{dim}} \mathrm{NK}$ cells produced variable responses because immature NK cells were preferentially being primed. We tested this hypothesis using mass cytometry, which permitted simultaneous analysis of more than 30 parameters on each NK cell (Supplemental Table 1). Using viSNE (32), we clustered control or IL-15-primed NK cells stimulated with K562 tumor targets into CD56 $6^{\text {bright }}$, CD56 $6^{\mathrm{dim}}$, immature CD56 $6^{\mathrm{dim}}$, and mature CD56 ${ }^{\mathrm{dim}}$ subsets based on the expression of maturity markers that were unaffected by priming (KIR, NKG2A, CD57) as well as CD56 (Figure 2, A and B). Imma-

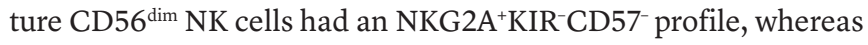
mature CD56 ${ }^{\text {dim }} \mathrm{NK}$ cells were primarily $\mathrm{NKG}^{2} \mathrm{~A}^{+} \mathrm{KIR}^{+} \mathrm{CD} 57^{+}$or $\mathrm{NKG}^{-} \mathrm{A}^{-} \mathrm{KIR}^{+} \mathrm{CD} 57^{+}$. The proportions of control or primed NK cells belonging to each CD56 ${ }^{\text {dim }}$ subset were similar (Figure $2 \mathrm{~A}$ ). Using mass cytometry to assess functional responses against K562 tumor targets, we found that cytokine (IFN- $\gamma$ and TNF) production 


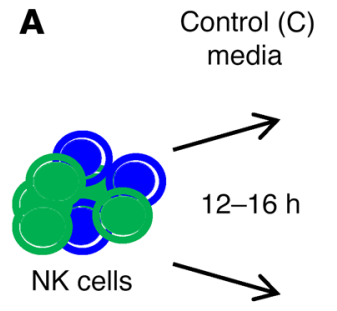

Primed $(\mathrm{P})$ media + IL-15
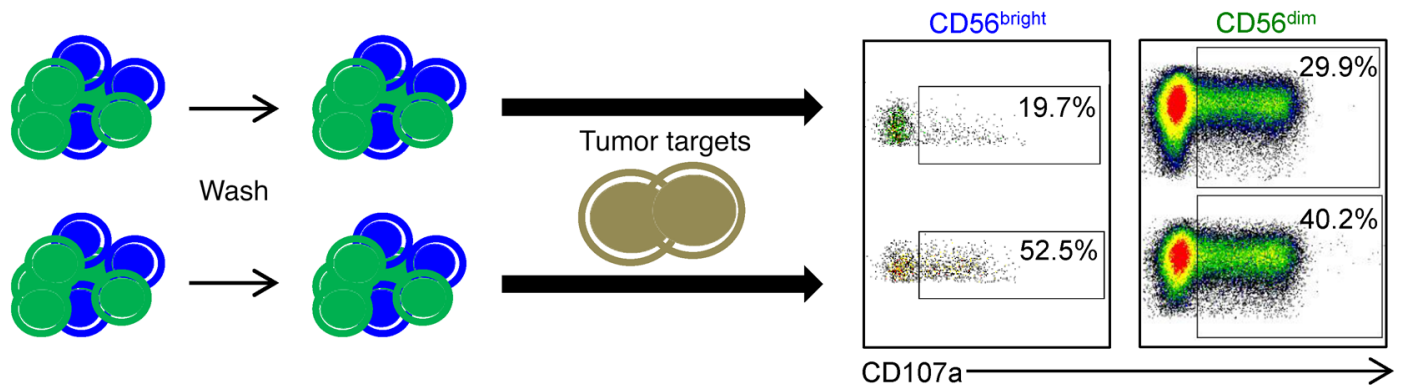

B
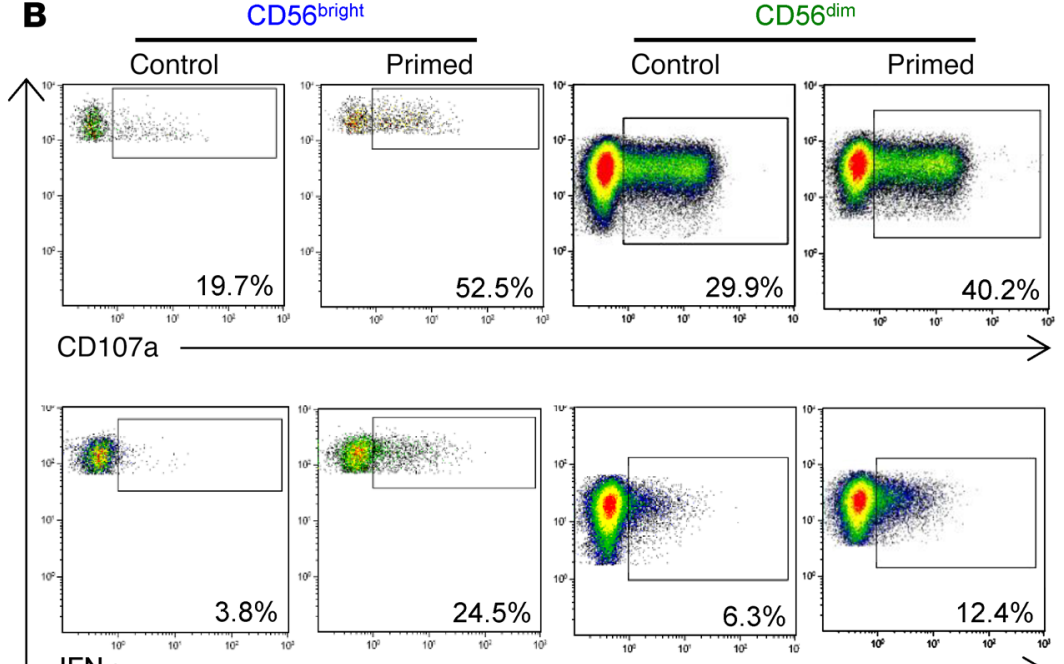

IFN- $\gamma$

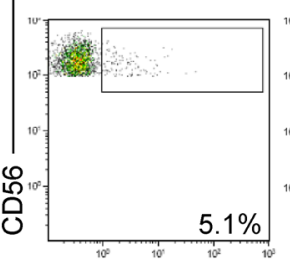

TNF

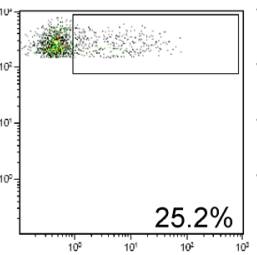

$25.2 \%$
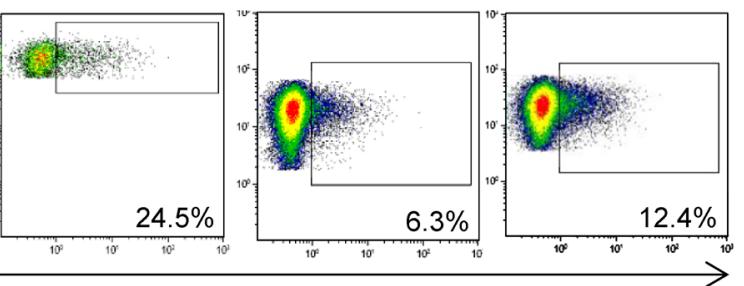
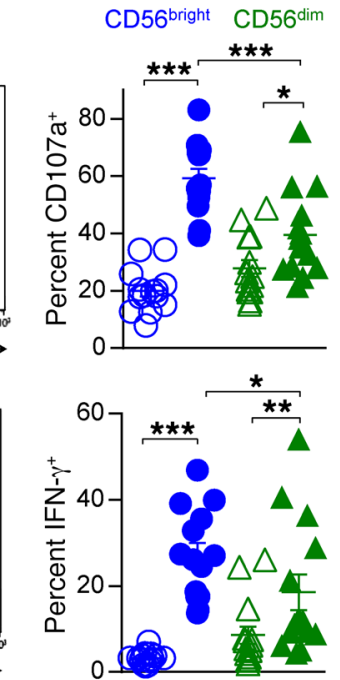

C $\mathrm{CD} 56^{\text {bright }} \mathrm{CD} 56^{\mathrm{dim}}$
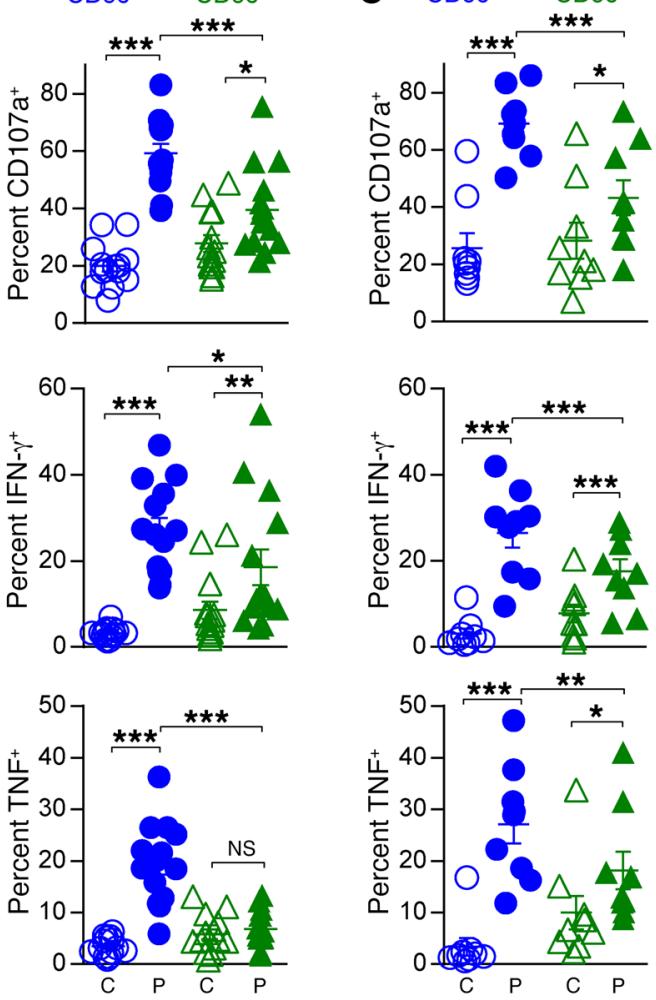

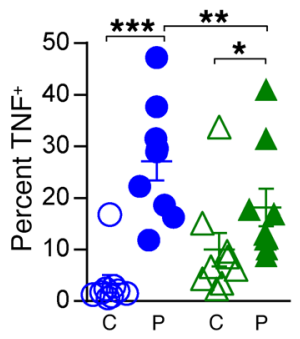

Figure 1. IL-15-primed human CD56 bright NK cells have enhanced responses to tumor target cells. (A) Purified NK cells from normal donors underwent short-term (12-16 hours) culture in media alone (control) or media with $5 \mathrm{ng} / \mathrm{ml} \mathrm{IL-15} \mathrm{(primed).} \mathrm{Cells} \mathrm{were} \mathrm{then} \mathrm{washed} \mathrm{and} \mathrm{triggered} \mathrm{with} \mathrm{tumor} \mathrm{targets.}$ (B) Control or primed purified NK cells were incubated with K562 AML target cells for 6 hours at a 5:1 E:T ratio. Bivariate flow cytometry plots from a representative normal donor show surface CD107a as well as intracellular IFN- $\gamma$ and TNF following tumor target triggering. Summary data show mean \pm SEM percentage of $\mathrm{CD} 107 \mathrm{a}^{+}$, IFN- $\gamma^{+}$, and TNF ${ }^{+} \mathrm{NK}$ cells. $n=14$ normal donors, 7 independent experiments. (C) Flow-sorted, control or primed CD56 bright and CD56 dim NK cells were triggered with K562 tumor targets for 6 hours at a 5:1 E:T ratio. Summary data show mean \pm SEM percentage of CD107a+, IFN- $\gamma^{+}$, and $\mathrm{TNF}^{+}$NK cells. $n=9$ normal donors, 6 independent experiments. Data were compared using a 1-way repeated-measures ANOVA, with Bonferroni's multiple-comparisons testing of indicated groups. ${ }^{*} P<0.05,{ }^{* *} P<0.01,{ }^{* * *} P<0.001$.

and degranulation by CD56 $6^{\text {bright }}$ NK cells were still significantly enhanced by priming; however, no significant differences were observed among CD56 ${ }^{\mathrm{dim}} \mathrm{NK}$ cells as a whole (Figure 2C). Degranulation and IFN- $\gamma$ production by immature CD $56^{\mathrm{dim}} \mathrm{NK}$ cells were significantly enhanced by IL-15 priming, but this increase was not seen in the mature CD56 ${ }^{\mathrm{dim}}$ subset (Figure 2C). We also compared the fold increase in primed (relative to control) NK cell subsets positive for CD107a, IFN- $\gamma$, or TNF upon K562 stimulation (Figure 2D). For all 3 functional markers, the fold increase was significantly higher for CD56 $6^{\text {bright }}$ than CD56 $6^{\mathrm{dim}} \mathrm{NK}$ cells, regardless of maturity status. Thus, IL-15 appears to preferentially prime immature CD56 $6^{\mathrm{dim}} \mathrm{NK}$ cells for enhanced degranulation and IFN- $\gamma$ pro- duction in response to K562 leukemia targets, but the magnitude of priming was less than for CD56 $6^{\text {bright }} \mathrm{NK}$ cells.

IL-15-primed CD56 $6^{\text {bright }} \mathrm{NK}$ cells display increased cytotoxic effector protein levels and enhanced cytotoxicity against tumor target cells. Since IL-15 priming markedly enhanced CD56 $6^{\text {bright }} \mathrm{NK}$ cell degranulation in response to tumor targets, we next evaluated cytotoxic protein expression and cytotoxicity. The perforin/ granzyme (granule exocytosis) pathway is a major mechanism used by NK cells to kill targets $(1,33)$. At rest, CD56 $6^{\text {bright }}$ NK cells express minimal perforin and granzyme $\mathrm{B}$ protein, and thus have low cytotoxic potential $(7,34)$. IL-15 priming significantly increased granzyme $\mathrm{B}$ and perforin protein levels in CD56 $6^{\text {bright }}$ 
A
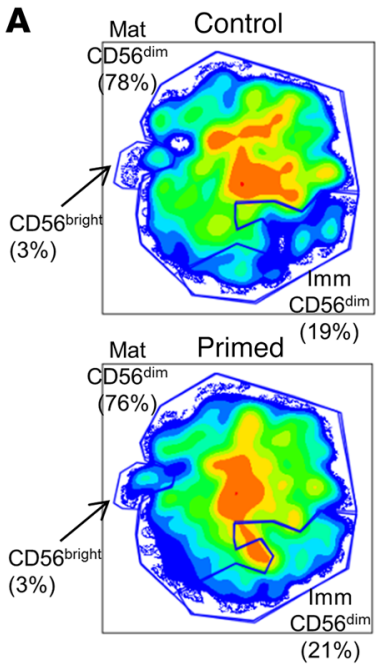

C
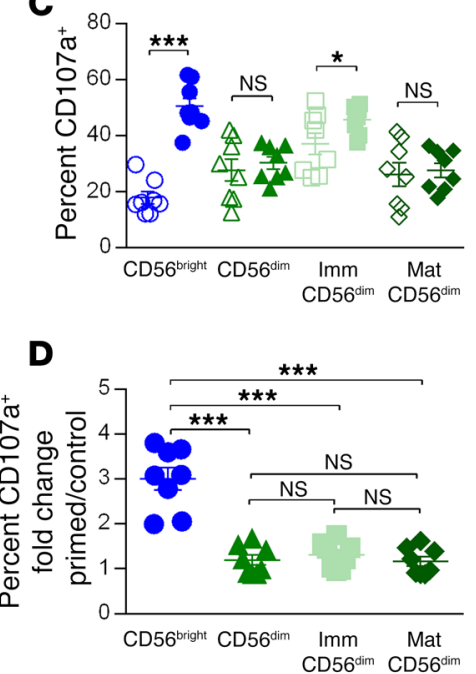

B
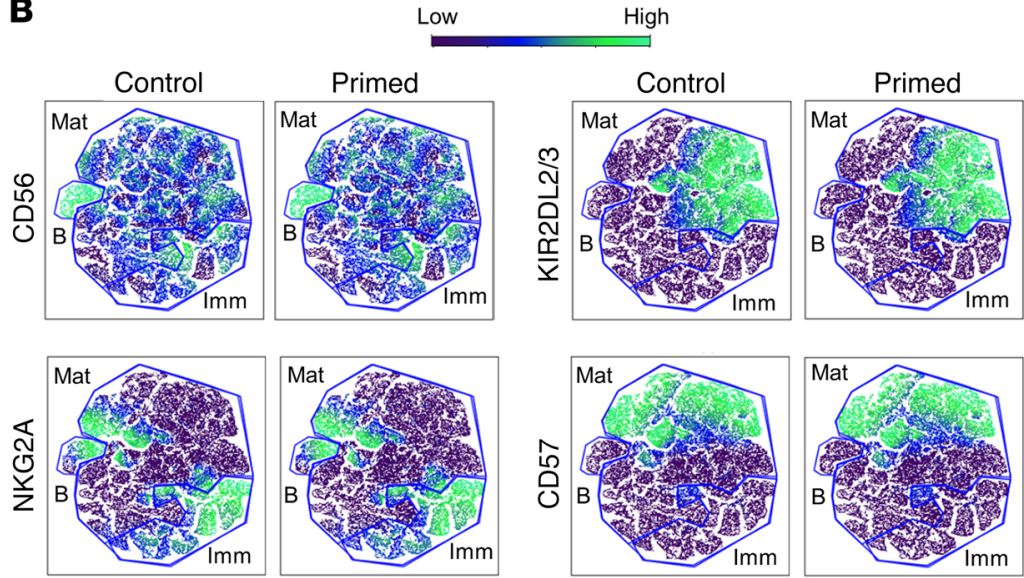
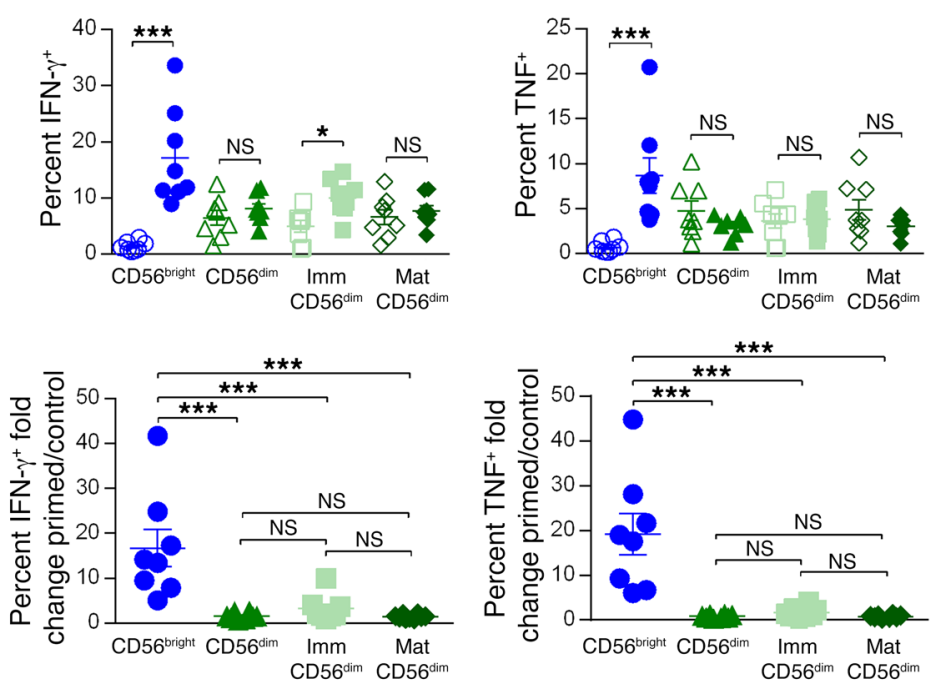

Figure 2. Responses of IL-15-primed CD56 ${ }^{\mathrm{dim}}$ NK cells are influenced by maturity status. Control and primed NK cells were assessed for the expression of 33 markers using mass cytometry following stimulation with $\mathrm{K} 562$ tumor targets in a functional assay. (A) Density plots from a representative donor of control and primed NK cells in the tSNE1/2 fields demonstrating the proportion of NK cells that are CD56 bright (B), immature CD56 dim (Imm), or mature CD56 dim (Mat). Percentages of NK cells belonging to the different groups are indicated in parentheses. Cell maturity state was determined according to expression of CD56, NKC2A, KIR, and CD57. Immature CD56 dim NK cells were primarily NKC2A+KIR-CD57-, whereas mature CD56 dim NK cells were primarily NKC2A+KIR+CD57+ or NKG2A-KIR+CD57+. (B) Median expression of the indicated markers (CD56, NKG2A, KIR2DL2/3, CD57) is shown for the same representative donor, demonstrating that maturity marker expression is unchanged by priming. (C) Summary data show mean \pm SEM percentage of CD107a+, IFN- $\gamma^{+}$, and TNF+ control or primed CD56 bright, CD56 dim (immature + mature), immature CD56 dim, and mature CD56 dim NK cells from $n=8$ normal donors, 3 independent experiments. (D) Summary data show mean \pm SEM fold change of primed relative to control percentage CD107a ${ }^{+}$, IFN $-\gamma^{+}$, and TNF CD56 $^{\text {bright }}$, CD56 ${ }^{\text {dim }}$ (immature + mature), immature CD56 dim, and mature CD56 dim NK cells from $n=8$ normal donors, 3 independent experiments. Data were compared using a 1-way repeated-measures ANOVA with (C) Bonferroni's multiple-comparisons testing of indicated groups or (D) Tukey's multiple-comparisons testing. ${ }^{*} P<0.05,{ }^{* * *} P<0.001$.

NK cells (Figure 3A). To directly assess the ability of IL-15 to augment cytotoxic function, flow-sorted CD $56^{\text {bright }}$ and CD $56^{\text {dim }} \mathrm{NK}$ cells were primed with IL-15 for 12-16 hours, and then assessed for cytotoxicity against K562 leukemia targets. IL-15 priming led to a substantial increase in the cytotoxicity of CD56 bright NK cells at all E:T ratios examined, but had a more modest impact on CD56 $6^{\mathrm{dim}}$ NK cell killing of K562 leukemia targets (Figure 3, $\mathrm{B}$ and $\mathrm{C}$ ). We also assessed expression of alternate mediators of NK cell target killing, such as the death receptor ligands TRAIL and Fas ligand. We observed a marked increase in TRAIL following IL-15 priming of CD56 $6^{\text {bight }} \mathrm{NK}$ cells (Figure 3A), but did not detect cell surface Fas ligand (not shown). The induced TRAIL is functional and contributes to IL-15-primed CD56 $6^{\text {bight }} \mathrm{NK}$ cell cytotoxicity, since killing of TRAIL-sensitive target cells (HL-60 AML and RPMI 8226 myeloma) was partially abrogated in the presence of TRAIL-neutralizing antibodies (Supplemental Figure 5 and refs. 35, 36). However, for relatively TRAIL-insensitive tumor targets such as U266 myeloma (35), TRAIL blockade did not substantially impact killing by IL-15-primed CD56 $6^{\text {bright }} \mathrm{NK}$ cells (Supplemental Figure 5).

To further define changes in CD56 ${ }^{\text {bright }}$ NK cells that may impact cytotoxicity, the granular area and granzyme B content of IL-15-primed CD56 $6^{\text {bright }}$ NK cells were assessed by immunofluorescence. At rest, CD56 $6^{\text {bright }}$ NK cells are small lymphocytes 

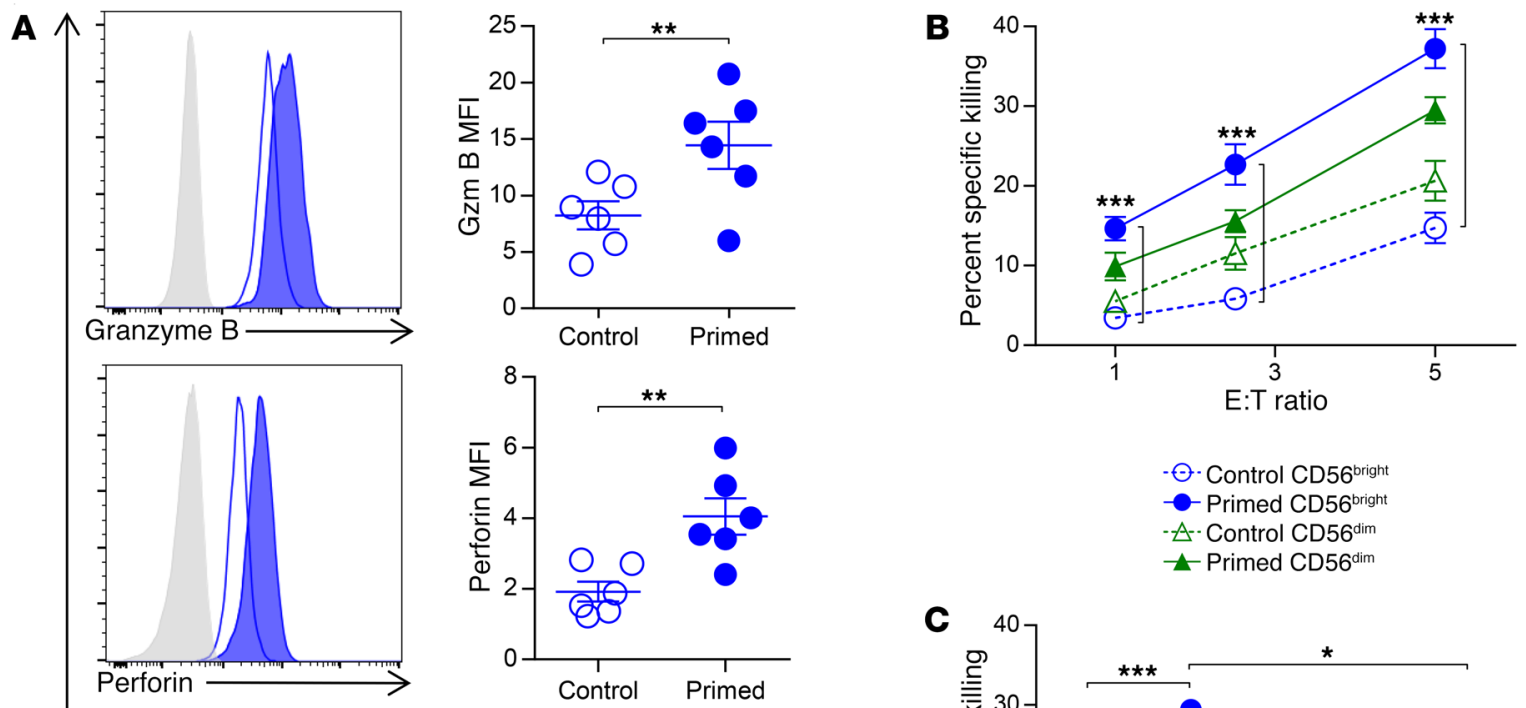

$$
\text { E:T ratio }
$$

-๑. Control CD56 bright

- Primed CD56 brigh

$\triangle-$ Control CD56 dim

- Primed CD56 dim
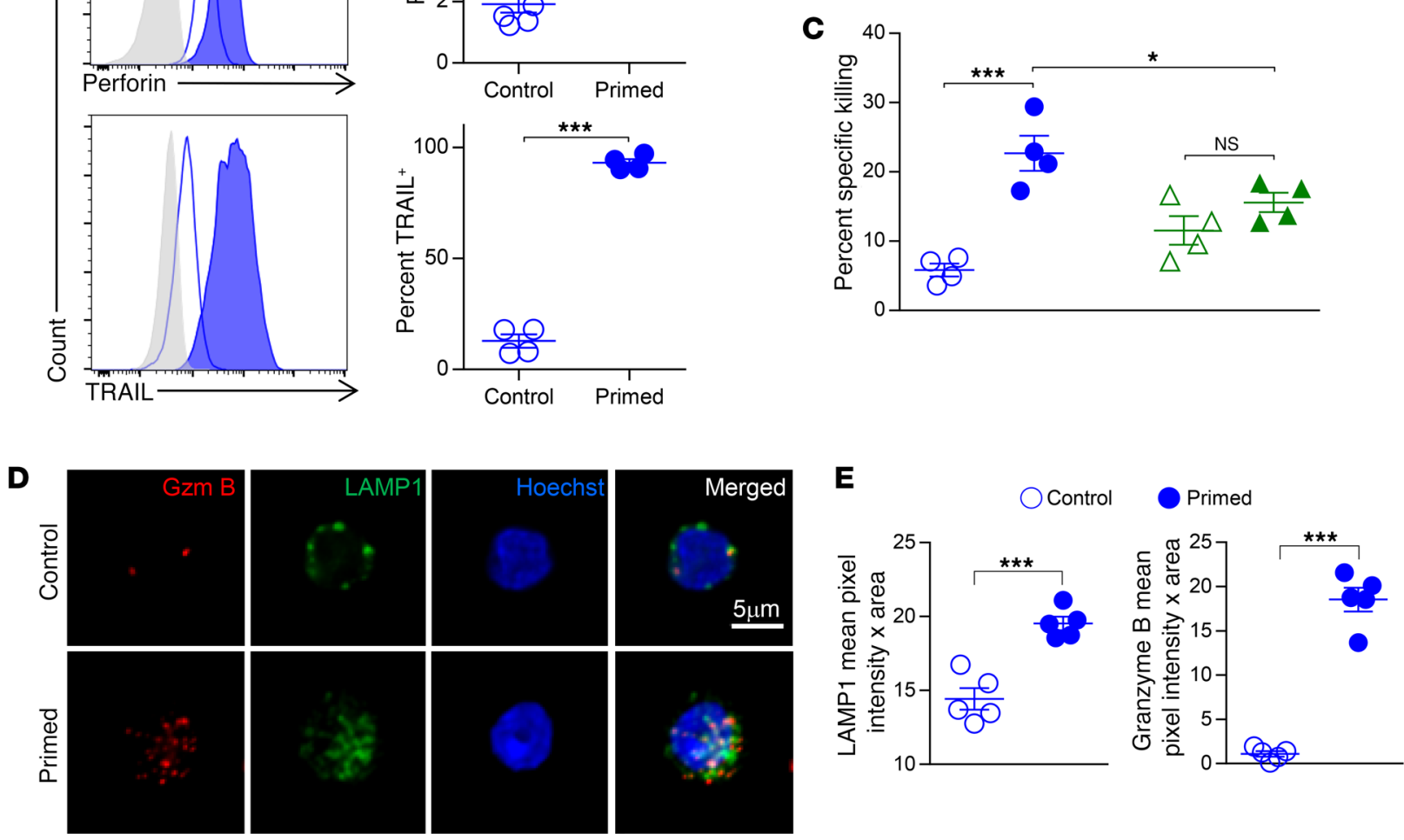

Figure 3. IL-15-primed CD56 bright NK cells have enhanced cytotoxicity. (A) Control or primed purified NK cells were assessed for expression of intracellular granzyme B and perforin and cell surface TRAIL using flow cytometry. Representative histograms gated on CD56 bright NK cells show per-cell protein expression, with gray histograms depicting unstained cells. Summary data show mean \pm SEM granzyme B and perforin median fluorescent intensity (MFI), or TRAIL percentage positive CD56 bright NK cells. $n=4-6$ normal donors, 2-3 independent experiments. (B and C) Control or primed flow-sorted CD56 bright and CD56 ${ }^{\text {dim }}$ NK cells were assessed for cytotoxicity against K562 leukemia cells in a 4-hour flow-based killing assay. (B) Summary data show mean \pm SEM percentage specific killing by control or primed NK cell subsets at the indicated E:T ratios. (C) Summary data show mean \pm SEM specific killing at the 2.5:1 E:T ratio. $n=4$ normal donors, 2 independent experiments. (D) Representative confocal Z-stack images show CD107a (LAMP1) and granzyme B (Czm B) in flow-sorted, control or IL-15-primed CD56 bright NK cells. Representative images were contrast-enhanced for better visualization. (E) Summary data show mean \pm SEM LAMP1 and granzyme B single-cell mean pixel intensity $\times$ area, quantified from focal planes in the middle of cells (control $n=20-32$, primed $n=25-37$ ) from 5 normal donors. Data were compared using (A and $\mathbf{E}$ ) a paired Student's $t$ test or $(\mathbf{B}$ and $\mathbf{C})$ a 1-way repeated-measures ANOVA, with Bonferroni's multiple-comparisons testing of indicated groups. ${ }^{*} P<0.05$, ${ }^{* *} P<0.01,{ }^{* *} P<0.001$.

that have few cytotoxic granules. IL-15 priming of CD56 bright $\mathrm{NK}$ cells led to markedly increased CD107a (LAMP1) and granzyme B mean pixel intensity as assessed by confocal immunofluorescence microscopy (Figure 3, D and E). This enhanced granularity of primed CD56 $6^{\text {bright }} \mathrm{NK}$ cells was also evident as an increase in morphologic cytoplasmic azurophilic granule content (Supplemental Figure 6A) and side scatter (Supplemental Figure 6B), neither of which was changed in CD56 $6^{\text {dim }}$ NK cells. Thus, IL-15-primed
CD56 ${ }^{\text {bright }}$ NK cells exhibit marked enhancement of multiple antitumor responses, including cytotoxic capacity and killing in vitro.

IL-15 priming promotes CD56 bright $N K$ cell conjugate formation with tumor targets. The NK cell cytotoxic response to tumor targets involves several events, including cell recognition, conjugate formation, immune synapse formation, and directed delivery of cytotoxic granule contents (33). We evaluated the effect of IL-15 priming on the efficiency of tumor conjugate formation by coin- 
A
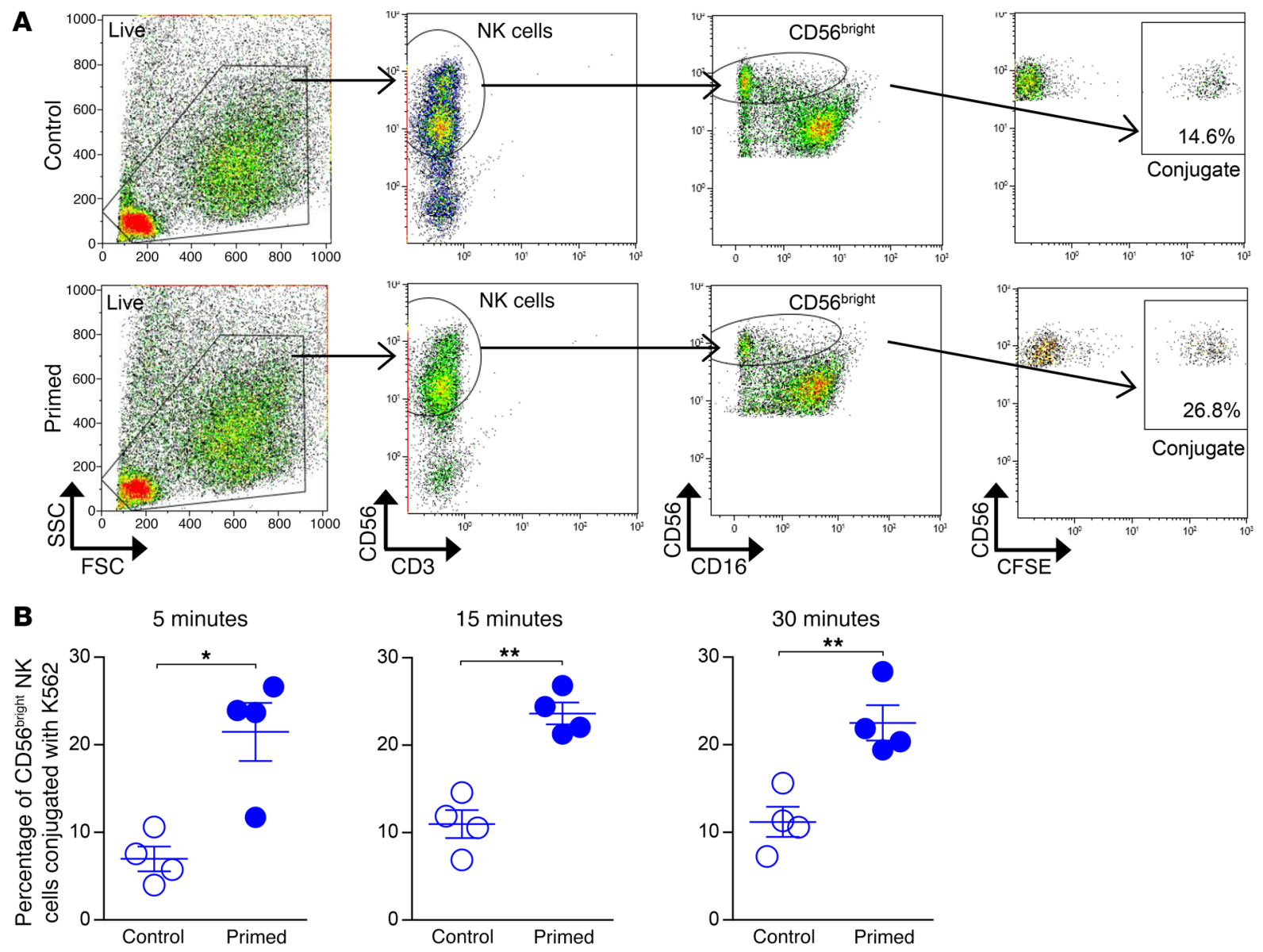

Figure 4. IL-15-primed CD56 bright $\mathbf{N K}$ cells more effectively form conjugates with K562 leukemia targets. Control or primed purified NK cells were assessed for conjugate formation via coincubation with CFSE-labeled K562 cells for 5, 15, or 30 minutes at a 1:1 E:T ratio. The NK/K562 conjugate percentage was assessed by the frequency of CD56 bright NK cell events that were also CFSE positive. (A) Representative bivariate flow plots show conjugate formation by control or primed NK cells at the 15 -minute time point by gating on (i) all live NK and K562 cells, (ii) NK cells (CD56+CD3'), (iii) CD56 bright NK cells, and (iv) CFSE-positive cells. (B) Summary data show mean \pm SEM percentage of control or primed CD56 bright NK cells that formed NK/K562 conjugates after 5 , 15 , and 30 minutes of coincubation. $n=4$ normal donors, 2 independent experiments. Data were compared using a paired Student's $t$ test. ${ }^{*} P<0.05,{ }^{* *} P<0.01$.

cubating control and primed NK cells with CFSE-labeled K562 cells for 5, 15, or 30 minutes (Figure 4A). A significantly greater percentage of IL-15-primed CD56 $6^{\text {bright }} \mathrm{NK}$ cells formed conjugates with tumor targets at each time point investigated compared with controls (Figure 4B). In contrast, there was no significant difference in the number of tumor conjugates formed by control versus IL-15-primed CD56 ${ }^{\text {dim }}$ NK cells at 5 minutes $(28.9 \% \pm 5.7 \%$ vs. $31.6 \% \pm 4.6 \%, P=0.053), 15$ minutes $(34.9 \% \pm 8.8 \%$ vs. $28.9 \% \pm$ $5.5 \%, P=0.17)$, or 30 minutes $(41.6 \% \pm 9.4 \%$ vs. $36.8 \% \pm 9.2 \%, P=$ $0.16)$ from the same donors. Thus, one mechanism underlying the enhanced antitumor response of IL-15-primed CD56 ${ }^{\text {bright }}$ NK cells involves more effective target recognition or target-cell synapse formation. Therefore, we next examined expression of adhesion molecules and NK cell receptors involved in tumor target recognition with or without IL-15 priming.

IL-15-primed CD56 bright $N K$ cells express more activating receptors and adhesion molecules than control cells. NK cells integrate adhesion molecule, activating and inhibitory receptor, and costimulatory signals, among others, to define their functional responses to target cells (3). We evaluated the surface expression of numerous such signaling molecules by flow cytometry. Expression of the fol- lowing proteins (percentage positive or median fluorescent intensity) did not differ between control and IL-15-primed CD56 bright NK cells: CD226 (DNAM-1), CD244 (2B4), NKp80, CD94, NKG2A, NKG2C, and CD137 (41BB) (data not shown). In addition, we confirmed no change in KIR (CD158a, CD158b1/2, CD158d, CD158e1/2, and CD158i) or CD57 expression on CD56 bright $\mathrm{NK}$ cells following IL-15 priming. However, we did observe significantly increased expression of NKG2D, NKp30, NKp44, CD69, $\mathrm{CD} 2$, and CD11a (Figure 5A and Supplemental Figure 7) on IL-15primed, compared with control, CD56 $6^{\text {bright }} \mathrm{NK}$ cells, in agreement with prior reports $(37,38)$. Because of their role in promoting antitumor responses by NK cells, NKG2D, NKp44, NKp30, CD2, and CD11a were thus evaluated for their contribution to IL-15-primed CD56 ${ }^{\text {bright }} \mathrm{NK}$ cell responses to tumor targets. We preincubated IL-15-primed NK cells with blocking mAbs against these receptors for 30 minutes, and then triggered the cells with K562 targets. No difference in $\mathrm{CD} 56^{\text {bright }} \mathrm{NK}$ cell degranulation or cytokine production upon NKp44 or NKp30 blockade was observed (data not shown). A small reduction in the priming response was observed when CD11a, CD2, or NKG2D was blocked individually. However, priming was significantly diminished upon simultaneous block- 
$\mathbf{A} \wedge$
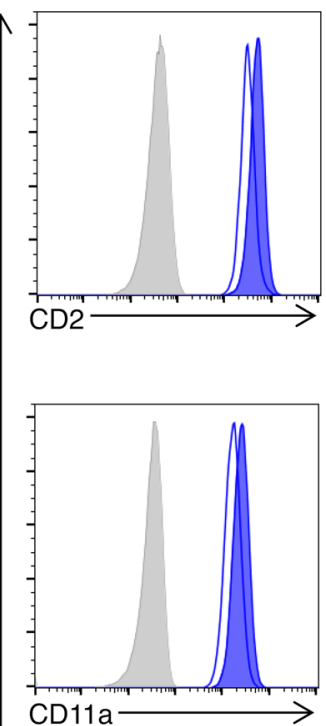

CD11a

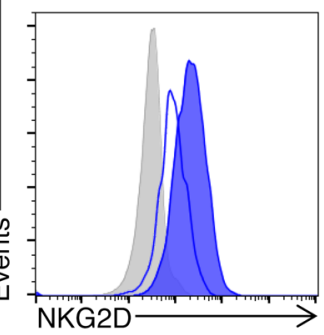

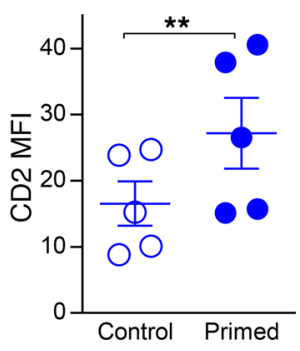
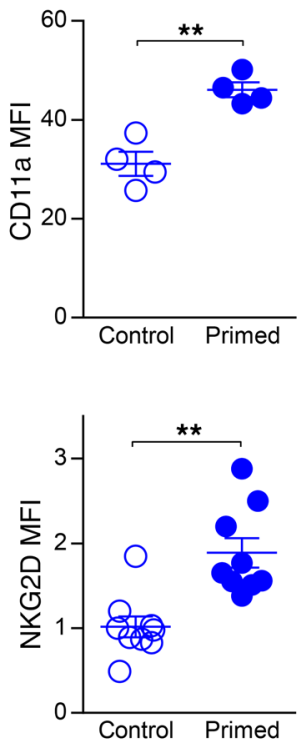

C
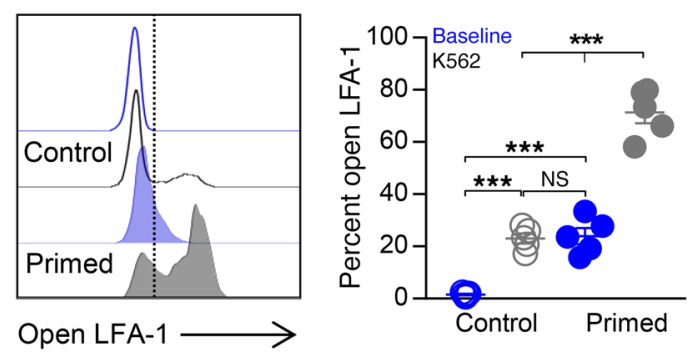
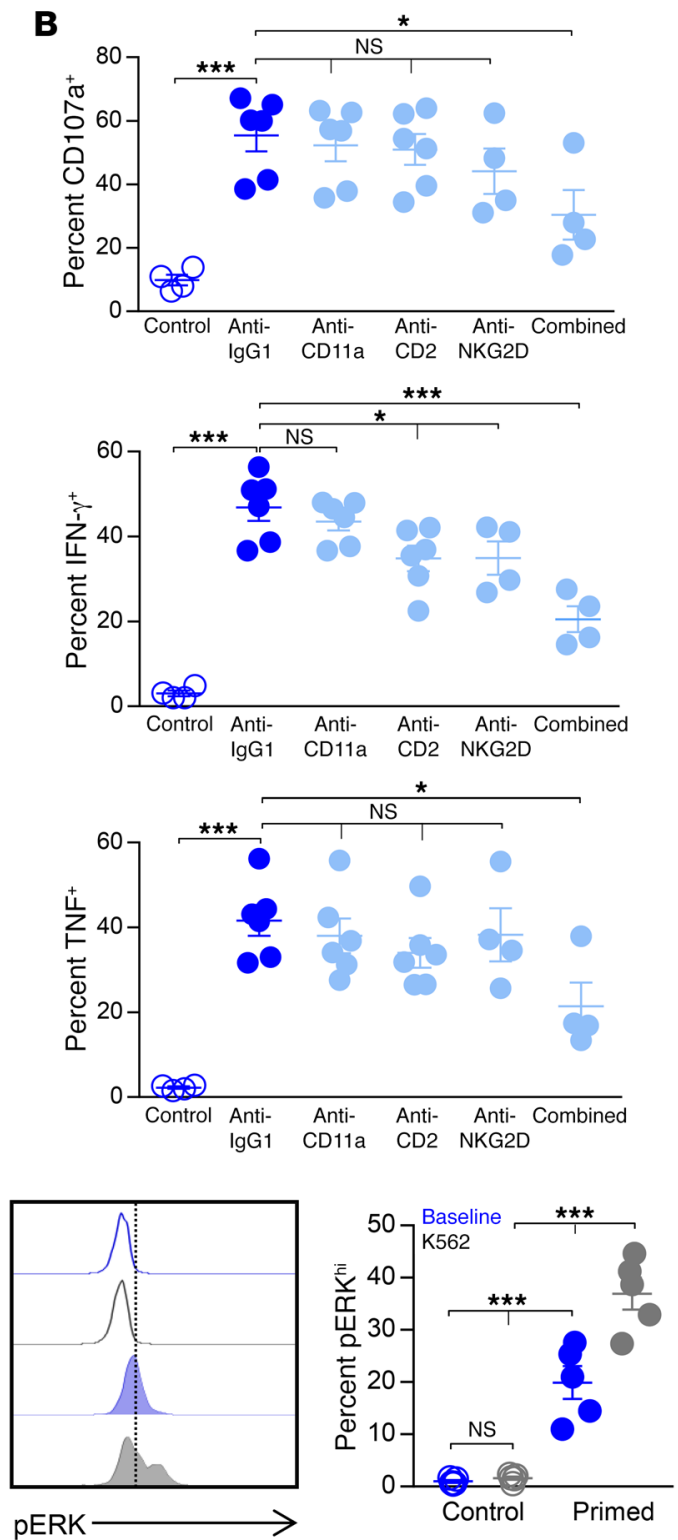

Figure 5. IL-15-primed CD56 bright $\mathrm{NK}$ cells display increased expression of coactivating and adhesion molecules as well as enhanced integrin signaling upon tumor target engagement. (A) Representative histograms gated on control or primed CD56 bight NK cells show per-cell surface expression of CD2, CD11a, and NKC2D, with gray histograms depicting unstained cells. Summary data show mean \pm SEM CD2, CD11a, and NKG2D MFI on control or primed CD56 bright NK cells. $n=4-9$ normal donors, 2-4 independent experiments. (B) IL-15-primed purified NK cells were preincubated with isotype control or blocking anti-human mAbs against CD11a, CD2, or NKG2D (or all 3 combined) for 30 minutes prior to coincubation with K562 target cells for 6 hours at 5:1 E:T ratio. Control NK cell responses were also assessed without blocking mAbs. Summary data show mean \pm SEM percentage of CD107a+, IFN- $\gamma^{+}$, and TNF ${ }^{+}$CD56 bright $N K$ cells. $n=4-6$ normal donors, 2-3 independent experiments. (C) Control or primed purified NK cells were assessed for the expression of open-conformation CD11a (mAb 24) or intracellular phosphorylated ERK (pT202/pY204) following 15 or 3 minutes (respectively) of coincubation with K562 tumor targets (K562, gray), or in the absence of targets (Baseline, blue). Representative histogram plots show per-cell open CD11a on CD56right NK cells. Summary data show mean \pm SEM percentage of control or primed CD56right NK cells expressing open CD11a (positivity cutoff set on baseline control cells). Representative histogram plots show per-cell pERK expression in CD56 bright NK cells. Summary data show mean \pm SEM percentage $p E R K^{\text {hi }}$ control or primed CD56 bright $N K$ cells ( $p E R K^{\text {hi }}$ cutoff set on baseline control cells). Data were compared using (A) a paired Student's $t$ test, (B) a 1-way ANOVA with Bonferroni's multiple-comparisons testing of indicated groups, or (C) a 1-way repeated-measures ANOVA with Tukey's multiple-comparisons testing. ${ }^{*} P<0.05,{ }^{* *} P<0.01,{ }^{* * *} P<0.001$.

ade of these 3 receptors (Figure 5B), indicating that they are collectively involved in the enhanced antitumor responsiveness of IL-15-primed CD56 ${ }^{\text {bright }} \mathrm{NK}$ cells.

IL-15 priming promotes integrin activation on $C D 56^{\text {bright }} \mathrm{NK}$ cells. CD11a and CD18 $\left(\beta_{2}\right)$ together constitute the integrin LFA-1. LFA-1 is critical for NK cell cytotoxicity; upon binding its ligands (the
ICAMs), LFA-1 initiates immune synapse formation and tight adhesion to target cells, and provides NK cell-activating signals (33, 39,40 ). Integrins typically exist in a bent conformation with low ligand-binding affinity. However, signals from activating receptors promote integrin activation via inside-out signaling, leading to high-affinity conformation changes and integrin clustering (41). 
NKG2D and CD2, among other molecules, can activate LFA-1 on NK cells (39). Thus, in addition to measuring overall LFA-1 (CD11a) expression, we also investigated the proportion of control or IL-15primed CD $56^{\text {bright }} \mathrm{NK}$ cells that expressed open-conformation LFA-1 at rest or following K562 target cell engagement. Incubation with IL-15 alone has been shown to only modestly activate LFA-1 on bulk NK cells; however, it enhances subsequent inside-out signaling following activating receptor ligation (42). Consistent with this, we observed a modest but significant increase in activated LFA-1 expression on CD56 $6^{\text {bright }} \mathrm{NK}$ cells as a result of IL-15 priming alone (Figure 5C). However, the majority of IL-15-primed CD56 $6^{\text {bright }} \mathrm{NK}$ cells expressed high-affinity LFA-1 after 15 minutes of incubation with $\mathrm{K} 562$ target cells, whereas K562 coincubation induced highaffinity LFA-1 on only a small subset of control CD56 $6^{\text {bright }} \mathrm{NK}$ cells (Figure 5C). Signaling through LFA-1 has been shown to activate ERK1/2 (43) (outside-in signaling), and enhanced ERK1/2 phosphorylation was observed in IL-15-primed CD56 $6^{\text {bright }} \mathrm{NK}$ cells trigged with K562 targets compared with control cells (Figure 5C). Thus, IL-15 priming promotes integrin activation on CD56 $6^{\text {bright }} \mathrm{NK}$ cells, potentially allowing adhesion to target cells in the absence of signals from other activating receptors (39), and also enhances subsequent target-induced inside-out and outside-in signaling.

IL-15 primes $C D 56^{\text {bright }} \mathrm{NK}$ cells from normal donors and $A M L$ patients for enhanced responses against primary AML blasts. We next examined whether IL-15 priming enhanced CD56 ${ }^{\text {bright }} \mathrm{NK}$ cell responses against primary leukemic blasts. Patient leukemia blast killing by flow-sorted, control or IL-15-primed CD56 $6^{\text {bright }}$ and CD56 ${ }^{\text {dim }}$ NK cells from allogeneic normal donors was examined (Figure 6A and Supplemental Table 2). IL-15 priming resulted in a significant increase in AML blast killing by CD56 $6^{\text {bright }}$, as well as CD56 $6^{\mathrm{dim}}$, NK cells (Figure 6A). In addition, since IL-15 is currently being tested in clinical trials for cancer therapy $(44,45)$, we investigated whether IL-15 could prime the endogenous CD $56^{\text {bright }}$ NK cells of patients with AML. Control or IL-15-primed peripheral blood mononuclear cells (PBMCs) from newly diagnosed AML patients were triggered with autologous AML blasts (Figure 6B) or K562 leukemia targets (Supplemental Figure 8A), and NK cell functional responses were assessed. Indeed, we observed significant enhancement of patient CD $56^{\text {bright }} \mathrm{NK}$ cell antitumor responses against both target cell types following IL-15 priming, with modest enhancement of CD56 $6^{\mathrm{dim}} \mathrm{NK}$ cell responses.

Next, the ability of IL-15-primed CD56 $6^{\text {bright }}$ NK cells to control leukemia in vivo was investigated by engrafting of groups of NOD-SCID-IL2R $\gamma^{-1-}$ (NSG) mice with either equal numbers of luciferase-expressing K562 cells (K562-luc) and ALT-803-primed CD56 ${ }^{\text {bright }} \mathrm{NK}$ cells, or K562-luc cells alone, via i.v. injection. ALT803 is an IL-15 superagonist complex exhibiting better pharmacodynamics and pharmacokinetic properties than recombinant IL-15 (46). ALT-803 is currently in clinical trials for cancer immunotherapy $(47,48)$. Both groups of mice received ALT-803 after cell engraftment (Figure 6C). We observed significantly lower tumor burden as assessed by whole-body bioluminescence imaging in mice treated with ALT-803-primed CD56 ${ }^{\text {bright }} \mathrm{NK}$ cells (Figure 6, D and E), demonstrating in vivo control of tumor cell growth by adoptively transferred, primed CD $56^{\text {bright }} \mathrm{NK}$ cells.

In vitro IL-15-primed CD56 $6^{\text {bright }} \mathrm{NK}$ cells show enhanced functional responses against multiple myeloma. To investigate whether the enhanced antitumor responses conferred by IL-15 priming extended beyond myeloid leukemia, we assessed responses of CD56 $6^{\text {bright }}$ NK cells against multiple myeloma (MM) targets. Enhanced degranulation and cytokine production by IL-15-primed, compared with control, CD56 $6^{\text {bright }} \mathrm{NK}$ cells from normal donors against U266 MM cells were observed (Figure 7A). We next tested whether IL-15 was able to prime newly diagnosed MM patient CD56 $6^{\text {bright }}$ NK cells for enhanced responses against autologous CD138 ${ }^{+} \mathrm{MM}$ cells (Figure 7B and Supplemental Table 3) or U266 MM targets (Supplemental Figure 8B). Indeed, degranulation and cytokine production were significantly increased in comparison with control CD56 ${ }^{\text {bright }}$ NK cells. Thus, in vitro responses against MM targets by allogeneic and autologous CD56 $6^{\text {bright }}$ NK cells were significantly improved after IL-15 priming.

ALT-803 primes CD56 ${ }^{\text {bright }} \mathrm{NK}$ cell antitumor responses in vivo in $M M$ patients. In addition, we investigated whether IL- 15 can prime NK cells in vivo using PBMCs from relapsed or refractory (rel/ ref) MM patients being treated with ALT-803 (NCT02099539). First, we verified that ALT-803 primed normal-donor CD56 $6^{\text {bright }}$ NK cell antitumor responses in vitro, similar to IL-15 (Supplemental Figure 9). We collected PBMCs from rel/ref MM patients just before, 24 hours after, or 72 hours after a single injection of ALT803 , and immediately assessed NK cell function in response to ex vivo stimulation with U266 myeloma target cells (patient group A) or the traditional NK-sensitive K562 tumor target (patient group B) (Figure 7C). Twenty-four hours after ALT-803 administration, patient CD56 $6^{\text {bright }} \mathrm{NK}$ cell degranulation and cytokine production in response to $\mathrm{MM}$ and leukemia target cells were significantly increased compared with pretherapy values (Figure 7D). However, this priming effect was relatively transitory in vivo, and responses no longer differed significantly from those observed before therapy by 72 hours after ALT-803. Thus, IL- 15 primed CD56 $6^{\text {bright }}$ NK cells both in vitro and in vivo for enhanced functional responses against MM target cells.

IL-15 more robustly activates the PI3K/Akt/mTOR and Ras/Raf/ $M E K / E R K$ pathways in CD56 bright $N K$ cells. Having identified elements responsible for the enhanced antitumor responsiveness of primed CD56 $6^{\text {bright }}$ NK cells, we next examined signaling mechanisms that could be responsible for priming of the CD56 $6^{\text {bright }}$ subset by IL-15. In mice, IL-15-induced activation of the PI3K/Akt/mTOR pathway is of particular importance for promoting NK cell cytotoxicity $(49,50)$, and mTOR activity downstream of the IL-15R has been shown to increase granzyme B expression in both murine and human NK cells (50). We confirmed that CD56 $6^{\text {bright }}$ and CD56 dim NK cells both robustly express IL-15R $\beta / \gamma$ (Figure 8, A and B). Next, the role that each pathway (JAK/STAT, PI3K/Akt/mTOR, and Ras/ Raf/MEK/ERK) downstream of the IL-15R plays in CD56 bright NK cell priming was investigated. Induction of each pathway (via phosphorylation of signaling intermediates) upon exposure to IL-15 in CD56 ${ }^{\text {bright }}$ and CD56 $6^{\mathrm{dim}} \mathrm{NK}$ cells was examined (Figure 8, D-F). IL-15 robustly induced phosphorylation of STAT5 in both CD56 ${ }^{\text {bright }}$ and CD56 ${ }^{\mathrm{dim}}$ NK cells (Figure 8D). In contrast, IL-15 more selectively induced Akt and ERK phosphorylation in CD56 $6^{\text {bright }}$ NK cells, with a minimal fold increase over baseline observed in the CD56 $6^{\mathrm{dim}}$ subset (Figure 8, E and F). This was not a matter of dose responsiveness, since even concentrations of IL-15 as high as $100 \mathrm{ng} / \mathrm{ml}$ did not enhance Akt and ERK phosphorylation in CD56 ${ }^{\text {dim }} \mathrm{NK}$ cells 

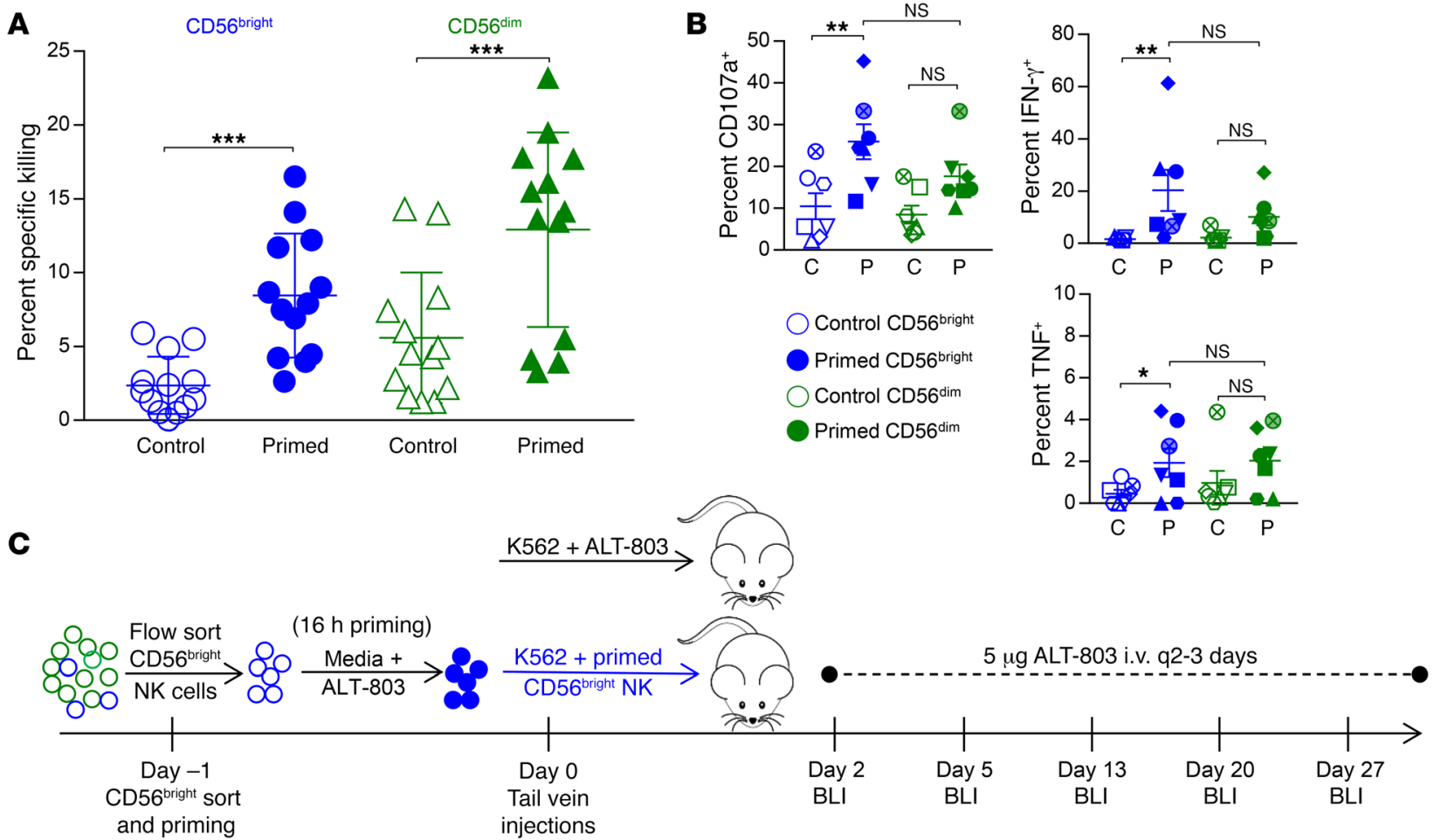

D

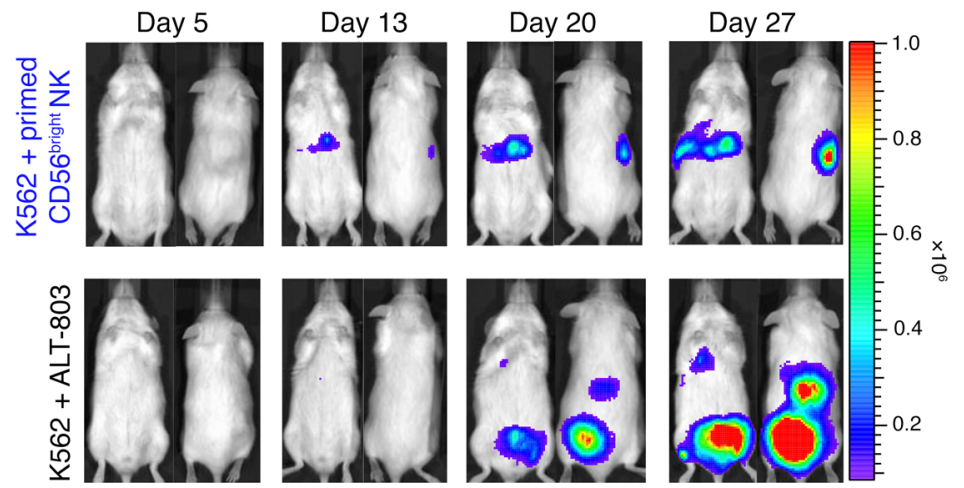

$\mathbf{E}$

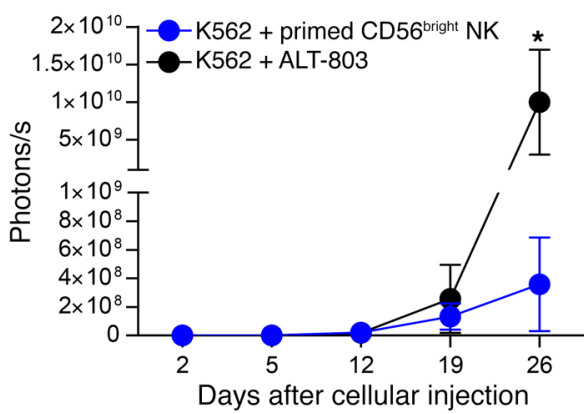

Figure 6. IL-15 priming enhances CD56 bright NK cell antitumor responses against primary AML blasts in vitro and K562 leukemia in vivo in NSG mice. (A) Flow-sorted, control or IL-15-primed CD56 bright and CD56 dim NK cells were coincubated with primary AML blasts from newly diagnosed patients. Summary data show mean \pm SEM percentage specific killing at a 15:1 E:T ratio. $n=7$ normal donors, 4 independent experiments. (B) PBMCs and bone marrow blasts were obtained from newly diagnosed AML patients. Control or IL-15-primed PBMCs were incubated with autologous blasts at a 5:1 E:T ratio for 6 hours and functional responses assessed. Summary data show mean \pm SEM percentages of CD107a+, IFN- $\gamma^{+}$, and TNF+ NK cells. $n=7$ normal donors, 3 independent experiments. (C) Experimental design. Day -1: Flow-sorted CD56 bright NK cells primed with $17.5 \mathrm{ng} / \mathrm{ml}$ ALT-803. Day 0: Primed CD56 bright NK cell injection into NSG mice. Eight mice received K562-Iuc $\left(0.5 \times 10^{6}\right.$ to $\left.0.66 \times 10^{6}, \mathrm{~K} 562+\mathrm{ALT}-803\right)$ and 8 mice received an equal number of both K562-luc and primed CD56 ${ }^{\text {bright }} \mathrm{NK}$ cells (K562 + primed CD56 bright NK). ALT-803 ( $5 \mu \mathrm{g}$ ) was administered i.v. on day 0 and every 2-3 days thereafter for 30 days. Bioluminescence imaging (BLI) was performed on days 2 , $5,13,20$, and 27, \pm 1 day. (D) Representative BLI images from one K562 + ALT-803 and one K562 + primed CD56bright NK mouse at different imaging time points. (E) Summary data of whole-body bioluminescence (photons per second) in the different treatment groups. Data show the mean \pm SEM photons per second at each time point. $n=8$ mice per group, 2 independent experiments. Data were compared using (A and $\mathbf{B})$ a 1-way repeated-measures ANOVA with Bonferroni's multiple-comparisons testing of indicated groups or (E) a 2-way ANOVA with Sidak's multiple-comparisons testing. ${ }^{*} P<0.05,{ }^{* *} P<0.01,{ }^{* * *} P<0.001$.

(data not shown). Since the PI3K/Akt/mTOR and Ras/Raf/MEK/ ERK pathways were more selectively induced by IL-15 in CD $56^{\text {bright }}$ NK cells, we hypothesized that they were important for priming and investigated this through pathway blockade.

The Ras/Raf/MEK/ERK pathway is required for IL-15-primed CD56 bright and CD56 ${ }^{\text {dim }} \mathrm{NK}$ cell responses, whereas the PI3K/Akt/ mTOR pathway is selectively involved in CD56 bright priming. We treated NK cells with PI3K (Ly294002) or MEK (PD98059) small- molecule inhibitors at different concentrations for 1 hour before priming with IL-15 and assessed the impact of this inhibition on functional responses to K562 targets (Figure 9, A and B). Inhibition of PI3K significantly impaired IL-15 priming of both degranulation and cytokine responses by CD56 $6^{\text {bright }}$ but not CD56 ${ }^{\mathrm{dim}} \mathrm{NK}$ cells in response to K562 leukemia targets (Figure 9A). Notably, impairment of IL-15 priming was not evident after mTOR inhibition by Torin 1 at concentrations that selectively inhibit mTORC1 
A
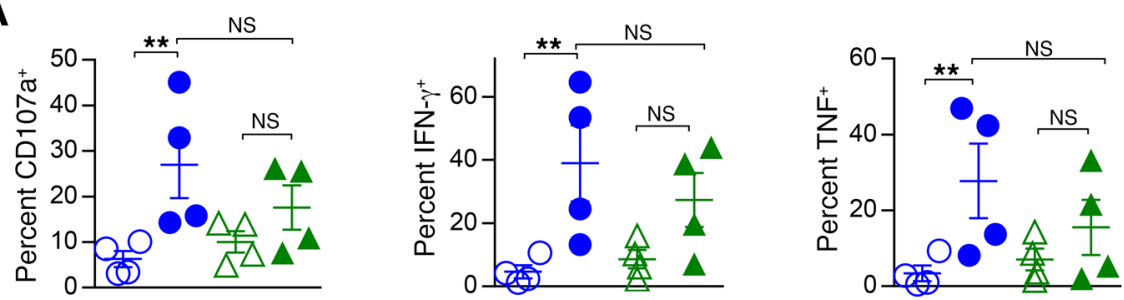

Control CD56brigh

Primed CD56 bright

$\triangle$ Control CD56 dim

$\triangle$ Primed CD56 dim
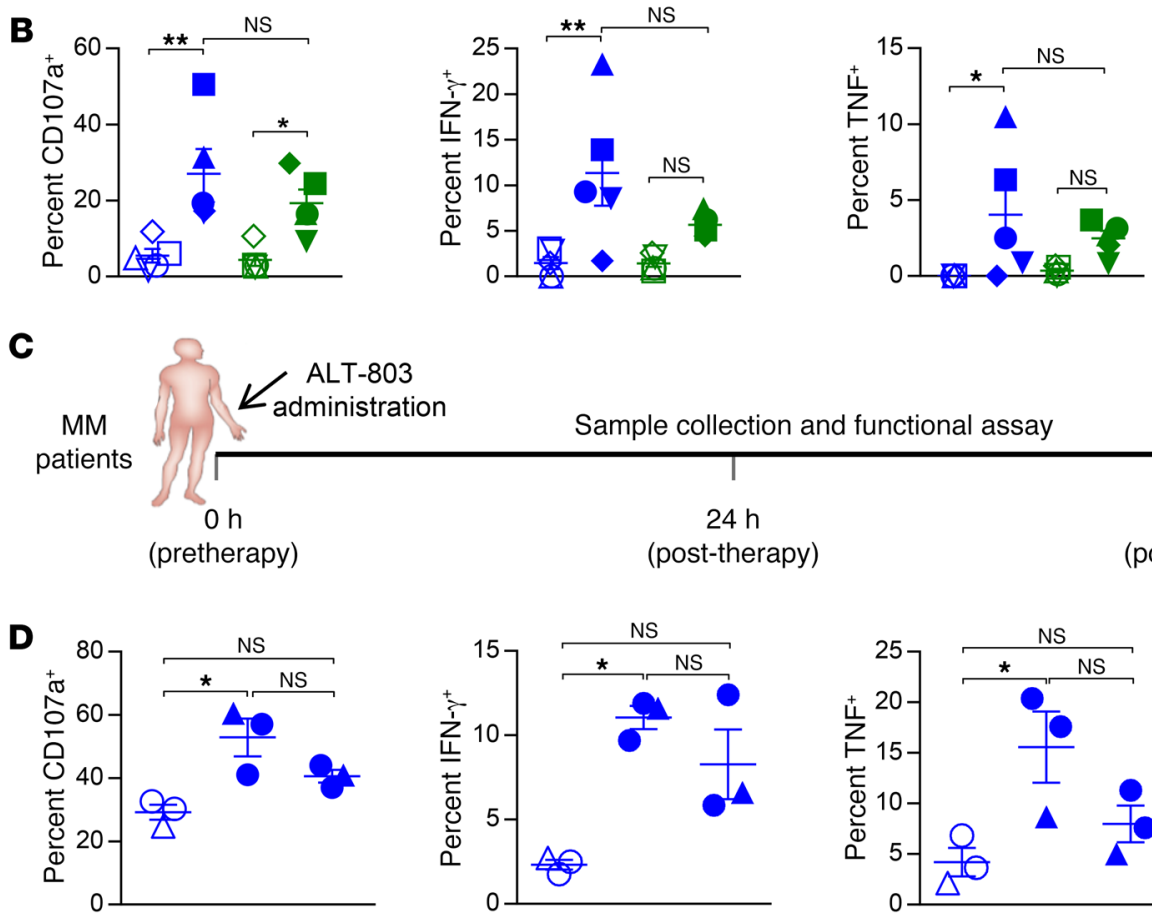

ALT-803
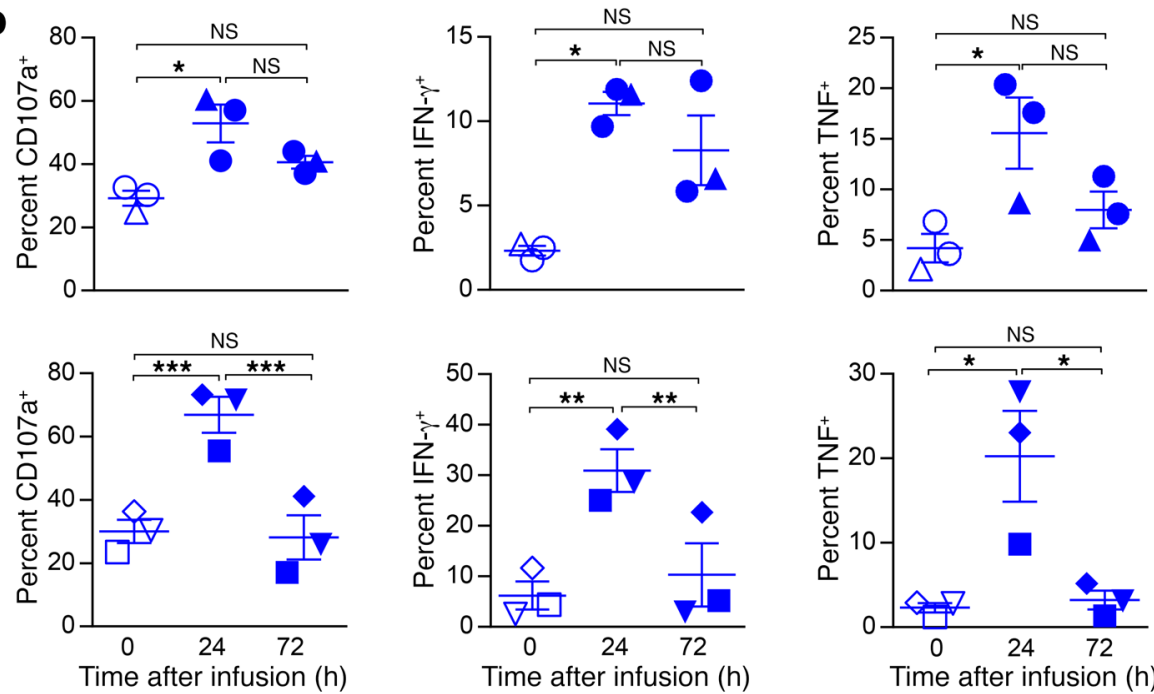

U266 MM

targets

(patient group A)
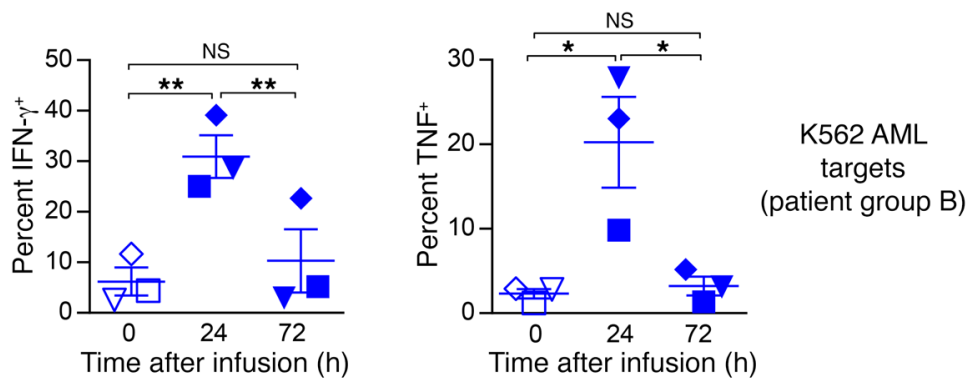

Figure 7. MM patient CD56bright NK cells are primed in vitro by IL-15 and in vivo by ALT-803 for enhanced functional responses against autologous myeloma cells and MM cell lines. (A) Control or IL-15-primed normal-donor purified NK cells were incubated with U266 MM target cells for 6 hours at a 5:1 E:T ratio. Summary data show mean \pm SEM percentage CD107a+, IFN- $\gamma^{+}$, or TNF ${ }^{+} N K$ cells. (B) PBMCs and enriched CD138 ${ }^{+}$cells were obtained from newly diagnosed MM patients. Control or IL-15-primed PBMCs were cultured with autologous CD138+ cells at a 5:1 E:T ratio for 6 hours and functional responses assessed. Summary data show mean \pm SEM percentage of $\mathrm{CD} 107 \mathrm{a}^{+}$, IFN- $\gamma^{+}$, and $\mathrm{TNF}^{+} \mathrm{CD} 56^{\text {bright }}$ or CD56 ${ }^{\text {dim }} \mathrm{NK}$ cells in response to autologous MM cell triggering. (C) Schematic of ALT-803 treatment regimen. Patients with rel/ref MM received $10 \mu \mathrm{g} / \mathrm{kg} \mathrm{ALT-803} \mathrm{s.c.} \mathrm{(group} \mathrm{A)} \mathrm{or} \mathrm{3-6} \mu \mathrm{g} / \mathrm{kg}$ i.v. (group B) at the 0-hour time point. Peripheral blood samples were obtained for functional analysis just before ALT-803 administration as well as 24 and 72 hours thereafter. (D) PBMCs were isolated from MM patient peripheral blood samples by Ficoll density centrifugation and immediately incubated with U266 myeloma targets (group A) or K562 leukemia targets (group B) for 6 hours. Summary data show mean \pm SEM percentage of CD107a+, IFN- $\gamma^{+}$, and TNF ${ }^{+}$CD56 bright $N K$ cells. $n=2$ patients, 3 independent experiments (group A); $n=3$ patients, 3 independent experiments (group B). Data were compared using 1-way repeated-measures ANOVA with (A and B) Bonferroni's multiple-comparisons testing of indicated groups or (D) Tukey's multiple-comparisons testing. ${ }^{*} P<0.05,{ }^{* *} P<0.01,{ }^{* * *} P<0.001$.

and mTORC2. However, higher concentrations that nonspecifically inhibit PI3K were able to abrogate IL-15 priming (Supplemental Figure 10 and ref. 51). MEK inhibition significantly impaired functional responses of both IL-15-primed CD56 $6^{\text {bright }}$ and CD56 $6^{\text {dim }}$ NK cells (Figure 9B). To confirm that the functional response abrogation conferred by PI3K or MEK inhibition was not an arti- fact of differential inhibitor affinity or E:T ratio, we repeated these experiments with flow-sorted CD56 $6^{\text {bright }}$ or CD $56^{\text {dim }}$ NK cells pretreated with both Ly294002 and PD98059. The same pattern of response was observed (Figure 9C and Supplemental Figure 11A). Furthermore, PI3K and MEK inhibition decreased cytotoxic protein expression in CD56 ${ }^{\text {bright }} \mathrm{NK}$ cells following IL-15 priming (Fig- 

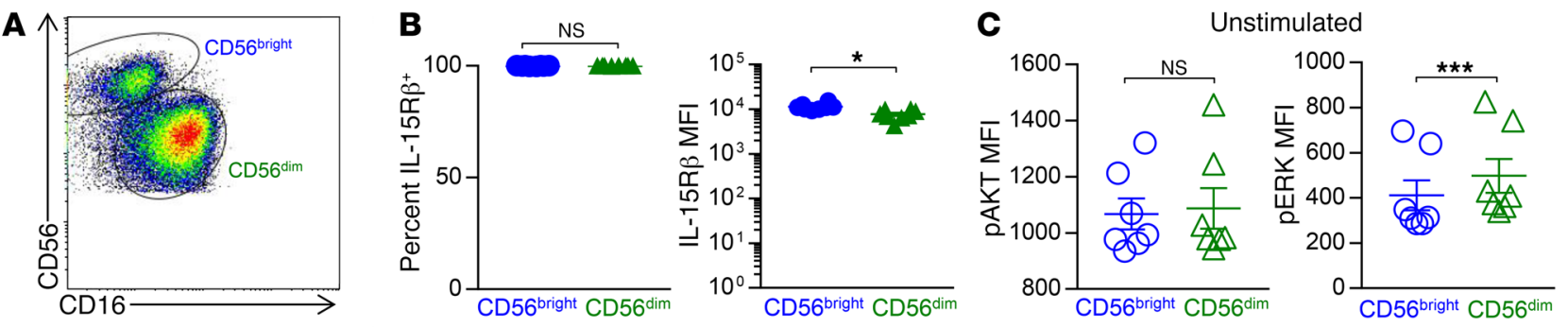

D

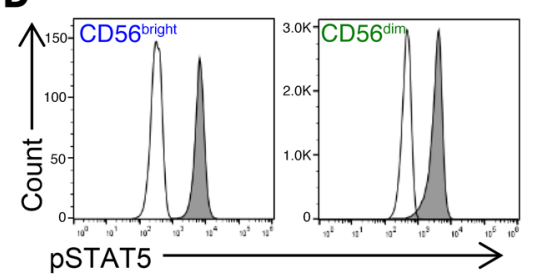

$\mathbf{E}$
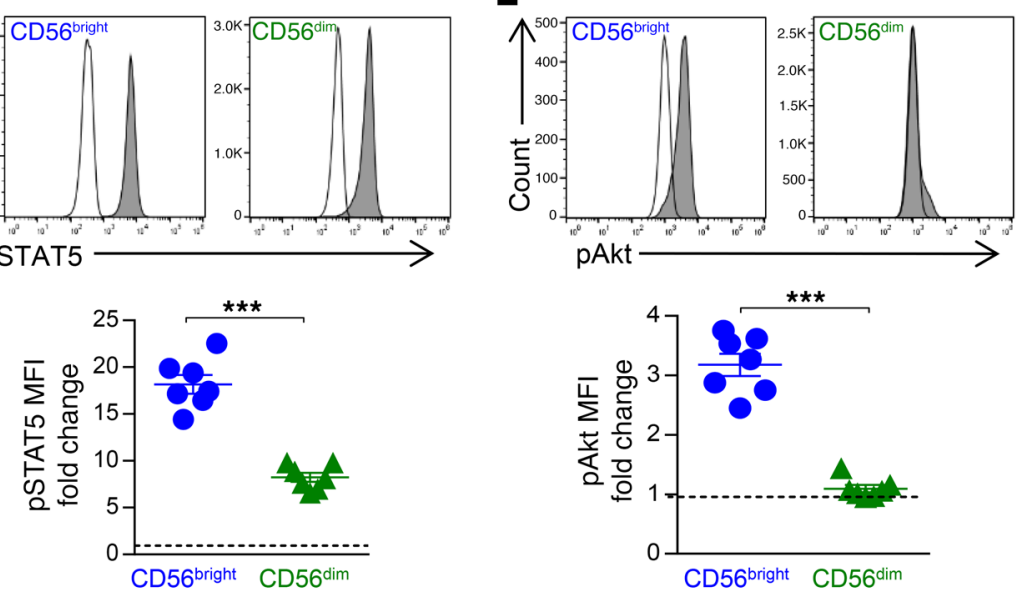

$\mathbf{F}$
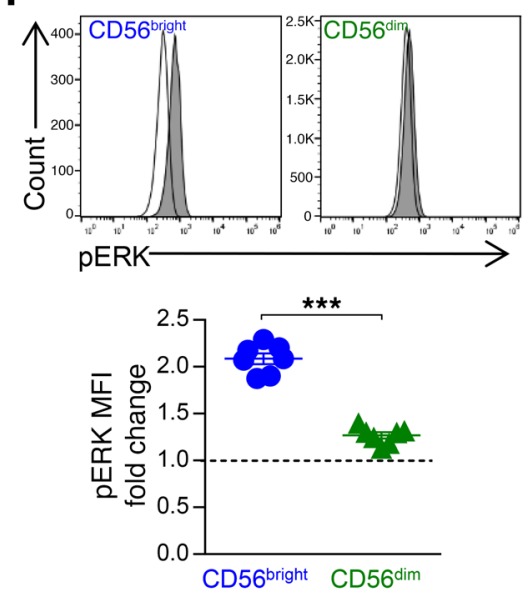

Figure 8. IL-15 more robustly activates the PI3K/Akt/mTOR and Ras/Raf/MEK/ERK pathways in CD56bright NK cells. (A) Flow cytometry plot shows gating strategy for CD56 bright versus CD56 ${ }^{\text {dim }}$ NK cells based on relative CD56 and CD16 expression. (B) IL-15R $\beta$ expression was assessed via flow cytometry on purified NK cells. Representative data show percentage IL-15R $\beta$-positive cells and IL-15R $\beta$ MFI on freshly purified CD56 bright and CD56 dim NK cells. $n=8$ normal donors, 3 independent experiments. (C) Summary data show pAkt and pERK MFI in unstimulated CD56 bright and CD56 dim NK cells. $n=7$ normal donors, 3 independent experiments. (D-F) Purified NK cells were incubated with $5 \mathrm{ng} / \mathrm{ml} \mathrm{IL-15}$ for 30 minutes (pSTAT5) or 2 hours (pAkt and pERK) then assessed for signaling molecule phosphorylation. Representative histograms show per-cell expression of pSTAT5 (D), pAkt (E), and pERK (F) in IL-15-stimulated (shaded gray) versus unstimulated (gray line) CD56 bright or CD56 ${ }^{\text {dim }}$ NK cells. Summary data show mean \pm SEM fold increase of phosphorylated molecule MFI in IL-15-stimulated CD56 ${ }^{\text {bright }}$ or CD56 ${ }^{\text {dim }}$ NK cells relative to unstimulated cells. $n=7$ normal donors, 3 independent experiments. Data were compared using a paired Student's $t$ test. ${ }^{*} P<0.05,{ }^{* *} P<0.001$.

ure 9D), and significantly reduced killing of K562 leukemia target cells (Figure 9E). While the effect of PI3K and MEK inhibition on CD56 ${ }^{\text {dim }}$ NK cell cytotoxic protein expression was more modest (Supplemental Figure 11B), K562 killing was significantly reduced as a result of treatment with these inhibitors (Supplemental Figure 11C). Thus, both the PI3K/Akt/mTOR and Ras/Raf/MEK/ERK pathways appear to be important for IL-15 priming of CD56 ${ }^{\text {bright }} \mathrm{NK}$ cells, while the PI3K/Akt/mTOR pathway was not required for the functional response of IL-15-primed CD56 $6^{\mathrm{dim}} \mathrm{NK}$ cells. Interestingly, our phosphorylation studies indicated that phosphorylated Akt (pAkt) expression is similar between unstimulated CD56 $6^{\text {bright }}$ and CD56 $6^{\text {dim }} \mathrm{NK}$ cells but pERK is significantly higher in CD56 $6^{\text {dim }}$ NK cells at baseline (Figure 8C). Thus, it is possible that a certain threshold of MEK/ERK pathway activation is required for significant antitumor responses, which is not achieved in nonprimed CD56 ${ }^{\text {bright }}$ NK cells or NK cells treated with PD98059.

\section{Discussion}

Numerous functional and phenotypic properties have been used to distinguish $\mathrm{CD} 56^{\text {bright }}$ from CD56 ${ }^{\text {dim }} \mathrm{NK}$ cells, including the superior ability of resting CD56 $6^{\text {dim }} \mathrm{NK}$ cells to be triggered by tumor targets or activating receptor ligation, and to mediate cytotoxicity. Here, we identified that brief priming through the con- stitutively expressed, intermediate-affinity IL-15R $\beta / \gamma$ markedly enhanced multiple antitumor functional responses (cytotoxicity, degranulation, and cytokine production) of CD56 ${ }^{\text {bright }}$ NK cells. These enhanced responses were elicited in vitro from allogeneic normal donor- and autologous cancer patient-derived NK cells triggered by primary AML blasts, primary myeloma cells, or a variety of tumor cell lines. IL-15 priming was also evident in vivo in patients with rel/ref MM treated with the IL-15 superagonist complex ALT-803, and in an NSG xenograft model where ALT803-primed CD56 bright NK cells significantly controlled K562 tumor burden. Several mechanisms contributed to the enhanced antitumor function of IL-15-primed CD56 $6^{\text {bright }} \mathrm{NK}$ cells, including increased cytotoxic protein levels, tumor conjugate formation, and CD2-, CD11a-, and NKG2D-based triggering. Thus, exposure to IL-15 unleashes the unexpectedly potent antitumor effector function of CD56 $6^{\text {bright }} \mathrm{NK}$ cells.

Cytokine activation of NK cells to promote antitumor responses has been explored in many clinical settings (52). Here, we examine short-term IL-15 activation, defined as the direct impact of IL-15 on the NK cell, and priming, the impact of IL-15 activation on subsequent antitumor responses. The effects of IL-15 priming on CD56 $6^{\text {bright }} \mathrm{NK}$ cells were transient, persisting for less than 72 hours after stimulation in vitro and in vivo in patients. This is dis- 
A

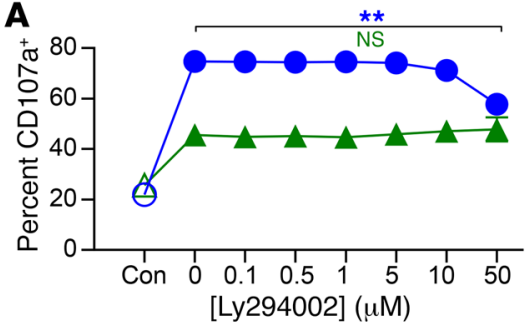

B

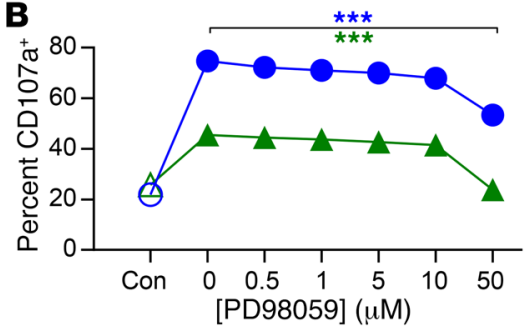

C
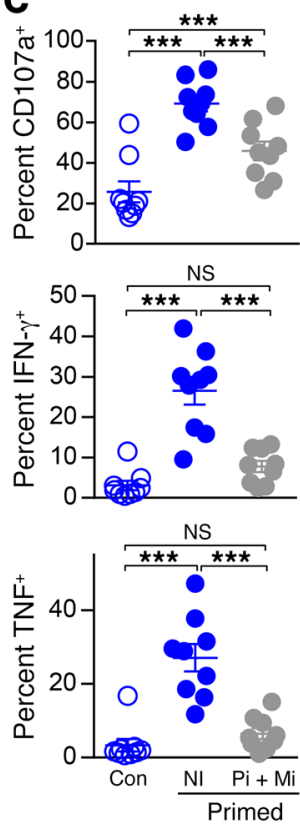

D
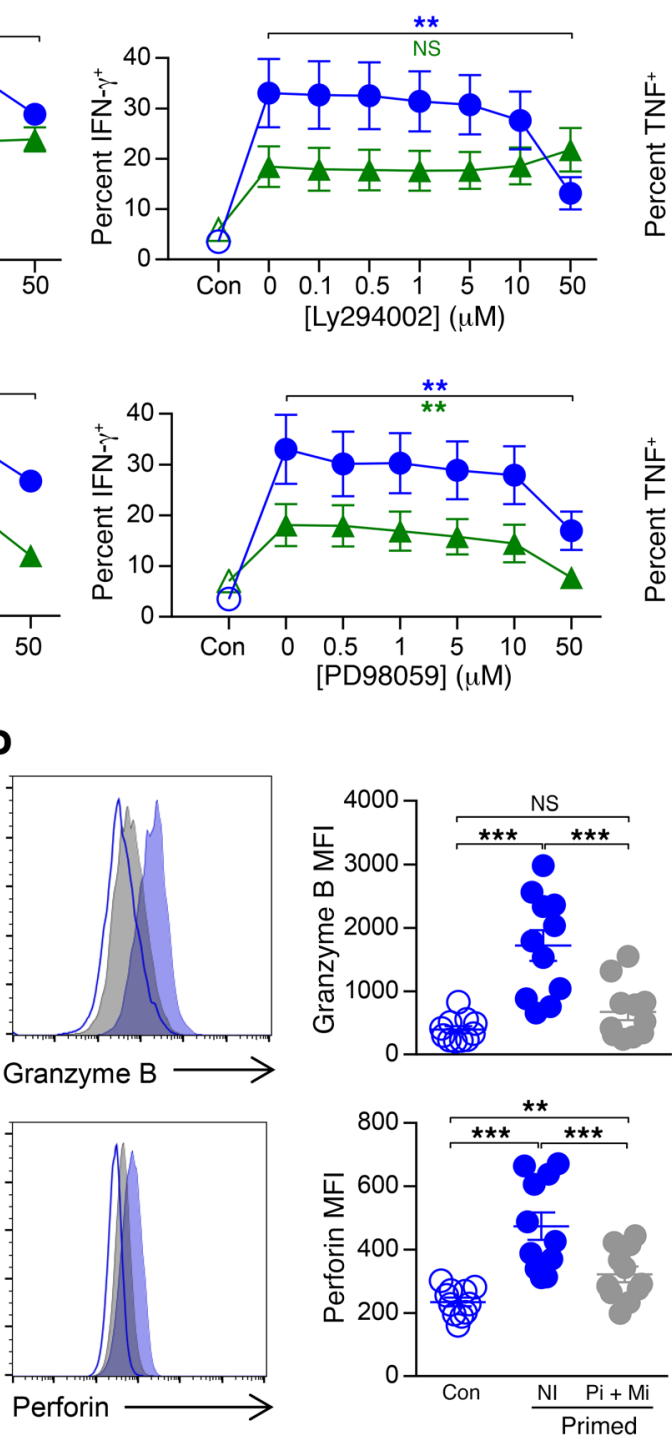

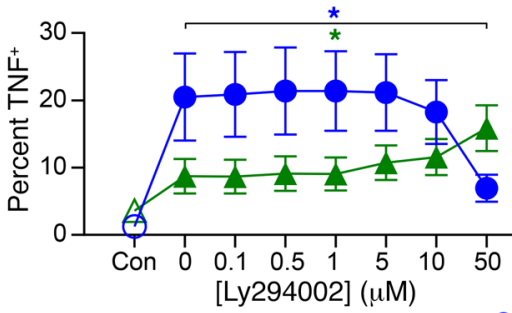

CD56 bright $\triangle \mathrm{CD} 56^{\mathrm{dim}}$

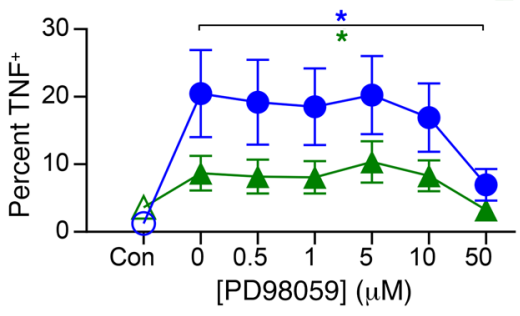

E

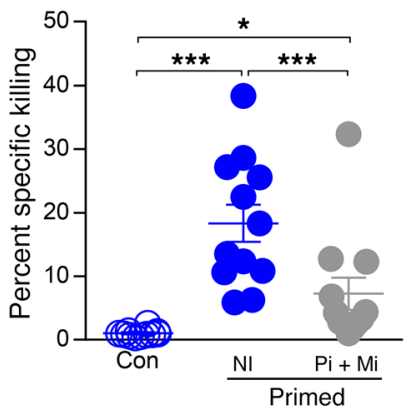

Control CD56 bright

Primed CD56 bright

Primed with PI3K and MEK inhibitors CD56 $6^{\text {bright }}$

Figure 9. The Ras/Raf/MEK/ERK pathway is required for IL-15 priming of CD56 bright and CD56 dim NK cell antitumor responses, whereas the PI3K/Akt/mTOR pathway is selectively required for CD56 bright $\mathbf{N K}$ cell priming. (A and B) Purified NK cells were cultured for $12-16$ hours in media alone (control, Con) or media plus $5 \mathrm{ng} / \mathrm{ml} \mathrm{IL-15} \mathrm{(primed).} \mathrm{In} \mathrm{addition,} 1$ hour before addition of IL-15, primed NK cells were treated with small-molecule inhibitors of PI3K (Ly294002) or MEK (PD98059) at various concentrations. Summary data show mean \pm SEM CD107a, IFN- $\gamma$, or TNF percentage positive control, primed, or primed in the presence of PI3K (A) or MEK (B) inhibitors CD56 bright and CD56 $6^{\text {dim }}$ NK cells. $n=8$ normal donors, 3 independent experiments. (C-E) Flow-sorted CD56 bright NK cells were cultured with or without (no inhibitor, NI) small-molecule inhibitors of PI3K (Ly294002, Pi) and MEK (PD98059, Mi) for 1 hour prior to 12-16 hours of IL-15 priming. Cells were then washed and incubated with K562 tumor targets for 6 hours at a 5:1 E:T ratio. (C) Summary data show mean \pm SEM CD107a, IFN- $\gamma$, or TNF percentage positive cells. $n=9$ normal donors, 6 independent experiments. (D) CD56 bright NK cells were assessed for granzyme B and perforin expression via intracellular flow cytometry. Representative histogram plots show per-cell cytotoxic protein expression. Summary data show mean \pm SEM granzyme B or perforin MFI. $n=11$ normal donors, 6 independent experiments. (E) CD56 bright NK cells were incubated with K562 tumor targets (E:T = 2.5:1) in a 4-hour flow-based killing assay. Summary data show mean \pm SEM percentage specific killing. $n=12$ normal donors, 8 independent experiments. Data were compared using (A and $\mathbf{B}$ ) a paired Student's $t$ test or (C-E) a 1-way repeated-measures ANOVA with Tukey's multiple-comparisons testing. ${ }^{*} P<0.05,{ }^{* *} P<0.01,{ }^{* * *} P<0.001$.

tinct from combined cytokine preactivation with IL-12, IL-15, and IL-18 that results in a prolonged enhancement of function (referred to as memory-like NK cell function) (53-56), and suggests that clinical approaches that use IL-15 priming of CD56 $6^{\text {bright }} \mathrm{NK}$ cells will likely require repeated IL-15 dosing to maintain primed status. Notably, our results demonstrating that IL-15 confers a selective survival advantage to CD56 ${ }^{\text {bright }} \mathrm{NK}$ cells suggest that these cells would survive preferentially over the $\mathrm{CD} 56^{\mathrm{dim}}$ subset in the setting of repeated ALT-803 administration, where drug concentrations may fall below therapeutic levels between doses. While CD56 $6^{\text {bright }}$ NK cells constitute a minor fraction of NK cells in the peripheral blood, they are the major subset in secondary lymphoid tissues, at sites of inflammation, and in some malignant tumors (9-11, 57, 58). Furthermore, NK cells reside predominantly in the $\mathrm{T}$ cell- 
and DC-rich parafollicular areas of secondary lymphoid tissues, and may normally be activated by DC-derived IL-15 (9-11). Prior reports demonstrated that CD56 $6^{\text {bright }} \mathrm{NK}$ cells from the peripheral blood, tonsils, and spleen were activated by mature DCs to more effectively prevent B cell transformation by EBV virus, compared with CD56 ${ }^{\text {dim }}$ NK cells (59). These data support the concept that CD56 $6^{\text {bright }} \mathrm{NK}$ cells may be primed in humans in vivo by IL-15 on the surface of DCs or monocytes in secondary lymphoid tissue (11), and potentially in other sites of IL-15 production such as the bone marrow and spleen $(60,61)$, thereby enhancing their functional competence against virally infected or malignantly transformed cells. Previous work has also revealed that exposure to IL-12 or prolonged stimulation with combinations of IL-2, IL-12, and/or IL-15 can increase CD56 ${ }^{\text {bright }} \mathrm{NK}$ cell cytotoxicity $(1,7,25,58,62$, 63). This prior work identified that CD56 $6^{\text {bright }} \mathrm{NK}$ cells may be cultured over days to weeks into lymphokine-activated killer cells that have increased potency against tumor cell lines. Our results are also consistent with a report evaluating CD56 $6^{\text {bright }} \mathrm{NK}$ cell responses to autologous activated $\mathrm{CD}^{+} \mathrm{T}$ cells, which were found to be enhanced by 4 -day culture with different cytokines, including IL-15 (64). Here, we report that brief cytokine priming via specific signaling pathways transforms immunoregulatory CD56 $6^{\text {bright }}$ NK cells into robust antitumor effectors exhibiting polyfunctional responses to a diverse set of cancer targets, both in vitro and in vivo. The robust increase in TRAIL expression of IL-15-primed CD56 ${ }^{\text {bright }} \mathrm{NK}$ cells furthermore suggests that these cells may also offer a substantial therapeutic benefit for TRAIL-sensitive tumors. Collectively, these studies highlight the plasticity of the CD56 bright NK cell subset, and indicate that cytokine cues from the NK cell microenvironment can profoundly alter its functional capacity.

The signaling mechanisms leading to IL-15 priming of CD56 ${ }^{\text {bright }}$ NK cells involve the PI3K/Akt/mTOR and Ras/Raf/ MEK/ERK pathways. These pathways were robustly induced by IL-15 in the CD56 $6^{\text {bright }}$ NK cell subset, and their blockade significantly impaired priming. This finding is consistent with studies investigating murine NK cell cytotoxicity (50), whereas reports concerning human NK cell cytotoxicity have been conflicting (38, $50,65)$, specifically with regard to the role of mTOR. Similar to reports from another group (38), we found that pretreatment of NK cells with the mTOR inhibitor Torin 1 at concentrations that selectively inhibit mTORC1/2 (10 nM) prior to priming did not impact effector responses (CD107a, IFN- $\gamma$, TNF) of CD56 $6^{\text {bright }}$ or CD56 ${ }^{\text {dim }}$ NK cells against K562 targets, or impair cytotoxic protein upregulation. Priming-induced increases in antitumor effector responses and cytotoxic protein levels were, however, impeded by Torin 1 at concentrations of $1 \mu \mathrm{M}$, similar to what has been used in other studies (65). However, micromolar concentrations result in off-target effects, including inhibition of PI3K (51). This supports a general PI3K/Akt/mTOR requirement for IL-15 priming of CD56 $6^{\text {bright }} \mathrm{NK}$ cells. Interestingly, only MEK/ERK pathway inhibition impacted IL-15 priming of CD56 ${ }^{\mathrm{dim}} \mathrm{NK}$ cells. Both the more robust PI3K/Akt/mTOR and MEK/ERK pathway activation in CD56 $6^{\text {bright }}$ compared with CD56 $6^{\text {dim }}$ NK cells by IL-15, and the selective involvement of the PI3K/Akt/mTOR pathway in CD56 ${ }^{\text {bright }} \mathrm{NK}$ cell priming, could then underlie the disproportionate enhancement of CD56 $6^{\text {bright }} \mathrm{NK}$ cell functional responsiveness by priming, in many situations to a level exceeding that of CD56 $6^{\mathrm{dim}}$
NK cells. The reasons for this differential responsiveness to IL-15 are unclear and warrant further investigation. Possibilities include SHIP1, which dephosphorylates the PI3K product PIP ${ }_{3}$, and has been shown to have lower expression in CD56 $6^{\text {bright }}$ than in CD56 ${ }^{\mathrm{dim}}$ NK cells, suggesting that this pathway is subject to less inhibition in the CD56 $6^{\text {bright }}$ subset (66). However, CD56 $6^{\text {bright }}$ NK cells have also been shown to express higher levels of PTEN, another inhibitor of the PI3K/Akt pathway (67). Additional studies linking IL-15R signals to distinct functions are warranted.

This study has translational relevance to several cancer immunotherapy settings, including the adoptive transfer of allogeneic NK cells $(17,68)$. Patients who receive allogeneic NK cell infusions are typically prepared with lymphodepleting chemotherapy, which has been shown to induce IL-15 protein in the serum (15, 68). Thus, in this situation, CD56 $6^{\text {bright }}$ NK cells that are present in the enriched NK cell product are likely primed in vivo by IL-15. Differences in the NK cell receptor biology of CD56 $6^{\text {bright }} \mathrm{NK}$ cells may also influence their antileukemic function. Since only a small fraction of CD56 $6^{\text {bright }} \mathrm{NK}$ cells express inhibitory KIRs, most inhibitory signals are mediated via CD94/NKG2A $(7,34)$. Thus, depending on their activation state, the CD56 $6^{\text {bright }}$ subset has the potential to respond to leukemia cells regardless of donor/recipient KIR/ KIR-ligand mismatch status. In our experiments, IL-15-primed CD56 ${ }^{\text {bright }}$ NK cells effectively responded to primary AML blasts without blocking CD94/NKG2A (or other inhibitory receptors). This suggests that IL-15 priming may alter the threshold of activation for CD56 ${ }^{\text {bright }} \mathrm{NK}$ cells, resulting in triggering by leukemia targets regardless of inhibitory signals. These findings are in line with reports of in vitro CD137L/IL-15-expanded bulk NK cells, for which activating receptor signals dominated inhibitory KIR signaling (69). Moreover, IL-15 or IL-15/IL-15R $\alpha$ complexes are being investigated in early-stage clinical studies to support adoptively transferred NK cells $(70,71)$ or as single agents $(45)$. Our findings suggest that the adoptively transferred or endogenous patient CD56 $6^{\text {bright }} \mathrm{NK}$ cells may contribute to the therapeutic efficacy of IL-15 administration. Finally, since ALT-803, unlike recombinant IL-15, accumulates and persists in secondary lymphoid tissues such as lymph nodes and spleen (72), treatment with this agent may be particularly apt to provide sustained priming signals to this lymph node-resident NK cell subset (9).

Another scenario in which IL-15 priming of CD56 $6^{\text {bright }} \mathrm{NK}$ cells may be important is early (1-3 months) after allogeneic stem hematopoietic cell transplantation (HCT). Early after an HCT, CD56 ${ }^{\text {bright }} \mathrm{NK}$ cells are the major lymphocyte subset to recover in the peripheral blood (73-78). Functional CD56 ${ }^{\text {bright }}$ NK cell antileukemic responses have not been analyzed in depth in this setting (57). In 2 reports, an "activated" phenotype with an intact CD56 ${ }^{\text {bright }}$ response to combined cytokine activation was observed $(76,78)$, although responses to leukemia or other target cells were not assessed. In a more recent study focused on $\mathrm{KIR}^{+} \mathrm{CD} 56^{\mathrm{dim}} \mathrm{NK}$ cells from HCT recipients, IL-15 stimulation led to a significant enhancement of the antileukemia responses of CD56 ${ }^{\mathrm{dim}}$ NK cells; however, the effects of IL- 15 on CD $56^{\text {bright }} \mathrm{NK}$ cell responses to leukemia cells were not reported (77). Based on our findings, elevated (or exogenous) IL-15 in the early postallogeneic HCT period may prime CD56 $6^{\text {bright }} \mathrm{NK}$ cells, and thereby promote NK cell clearance of residual AML blasts. Late after HCT, donor NK cells reestab- 
lish homeostasis after having developed from transplanted stem cells within the host, and are no longer being primed by excessive IL-15. At this time point, the more mature, educated $\mathrm{KIR}^{+} \mathrm{CD} 56^{\mathrm{dim}}$ NK cells would be expected to mediate longer-term antileukemia responses, consistent with well-studied associations between donor KIR genotype and transplant outcome (14, 20, 21).

In summary, CD56 $6^{\text {bright }} \mathrm{NK}$ cells, traditionally considered to be minimally tumor-responsive, are effectively primed by short-term IL-15 exposure to enable potent cytotoxicity, degranulation, and cytokine production in response to a broad array of tumor targets and primary hematologic tumor cells both in vivo and in vitro. This study provides a novel rationale for developing clinical immunotherapy approaches that utilize IL- 15 to prime CD $56^{\text {bright }}$ NK cells, and suggests that IL-15-based therapeutics may act in vivo to enhance the antitumor capacity of this NK cell subset.

\section{Methods}

Reagents. Antihuman mAbs used in this study are listed in Supplemental Table 5. Endotoxin-free rhIL-15 was used (Miltenyi Biotec). The following cell lines were maintained in RPMI-1640 plus $10 \%$ FCS and supplements: K562 (AML), HL-60 (AML), U266 (MM), and RPMI 8226 (MM). K562 cells were obtained from ATCC (CCL-243) in 2008, viably cryopreserved in liquid nitrogen, thawed for use in these studies, and maintained for less than 2 months at a time in culture according to provider instructions. K562 cells were authenticated in 2015 using SNP analysis and were found to be exact matches to the K562 cells from the Japanese Collection of Research Bioresources, German Collection of Microorganisms and Cell Cultures (DSMZ), and ATCC databases (2015, Genetic Resources Core Facility at Johns Hopkins University). Additional cell lines were obtained from ATCC and provided for our use by Daniel Link (HL-60) and Michael Tomasson (U266, RPMI 8226).

NK cell purification and cell culture. Anonymous human platelet apheresis donor PBMCs were obtained by Ficoll centrifugation. NK cells were purified using RosetteSep (StemCell Technologies; $\geq 95 \%$ $\mathrm{CD}^{+} 6^{+} \mathrm{CD}^{-}$) or by flow cytometric cell sorting (BD FACSAria II; $\geq 97 \%$ purity) (53). Cells were cultured in complete RPMI-1640 media containing 10\% human AB serum (Sigma-Aldrich) for 12-16 hours (unless otherwise stated) with or without $5 \mathrm{ng} / \mathrm{ml}$ rhIL-15. ALT-803 in vitro priming of NK cells was performed at $17.5 \mathrm{ng} / \mathrm{ml}$ for $12-16$ hours. For assays involving IL-15 signaling pathways, NK cells were preincubated with inhibitors of PI3K (Ly294002) or MEK (PD98059; both Cayman Chemical Co.) alone or in combination for 1 hour prior to priming.

Functional assays to assess degranulation and cytokine production. Normal-donor purified or flow-sorted CD56 bright and CD56 $6^{\mathrm{dim}} \mathrm{NK}$ cells were coincubated with $\mathrm{K} 562$ or other cell line targets (E:T ratio of 5:1) for 6 hours, with brefeldin A and monensin (BD Biosciences) added for the final 5 hours. Assessment of CD107a, IFN- $\gamma$, and TNF by flow cytometry was performed as previously described (53). In some experiments, NK cells were preincubated for 30 minutes with antiNKG2D, -CD2, -CD11a/LFA-1, -NKp44, or -NKp30 blocking mAbs $(10 \mu \mathrm{g} / \mathrm{ml})$ before $\mathrm{K} 562$ exposure. For ex vivo analysis of peripheral blood samples from MM patients receiving ALT-803, fresh PBMCs were isolated by Ficoll centrifugation and immediately coincubated with U266 myeloma ( $\mathrm{E}: \mathrm{T}=5: 1)$ or K562 leukemia ( $\mathrm{E}: \mathrm{T}=10: 1)$ targets, with functional readouts assayed as above. For assays involving in vitro priming of AML or MM patient NK cells, PBMCs were isolated by Ficoll centrifugation from newly diagnosed patients with relatively low peripheral blood disease burden and used fresh or viably cryopreserved. Control or primed patient PBMCs were then triggered with autologous bone marrow AML (bone marrow blast percentage $\geq 50 \%$ ) or enriched CD138 ${ }^{+}$MM cells, K562 leukemia cells, or U266 multiple myeloma cells ( $\mathrm{E}: \mathrm{T}=5: 1)$ with function assayed as above.

Proliferation and viability assays. Purified CFSE-labeled or unlabeled NK cells were incubated for 12-16 hours with media alone (control) or media with $5 \mathrm{ng} / \mathrm{ml} \mathrm{IL-15}$ (primed). Cytokines were then washed away, and cells were incubated in media alone for 3-5 days. To interrogate proliferation, after both 3 and 5 days, NK cells were harvested and surfacestained for NK cell markers, and CFSE dilution or intracellular Ki67 expression (BD Biosciences) was assessed via flow cytometry. To interrogate viability, after both 3 and 5 days, NK cells were surface-stained for NK cell markers, then stained for activated caspase- 3 and caspase-7 (CellEvent Caspase 3/7 Red, Thermo Fisher Scientific) and annexin V (BD) per the manufacturer's instructions. Baseline viability was determined using acridine orange and propidium iodide (Nexcelom).

Mass cytometry. Mass cytometry functional assays were performed as above. Sample staining and data collection were performed as previously described (56).

Flow-based killing assay. Flow-based killing assays were performed by coincubation of flow-sorted CD56 $6^{\text {bright }}$ or CD56 ${ }^{\text {dim }}$ NK cells with CFSE-labeled K562 cells or PKH67-labeled (Sigma-Aldrich) primary AML blasts for 4-5 hours and assaying of 7-AAD uptake as previously described (28). Background spontaneous K562 or AML blast death (no effector control wells) was subtracted to yield percentage specific killing, and in all cases this was less than $5 \%$ (K562) or 25\% (primary AML blasts). E:T ratios of 5:1, 2.5:1, and 1:1 were used for coincubation with K562 targets and 15:1 for primary AML blasts. For experiments investigating the contribution of TRAIL to target cell killing, flow-sorted, IL-15-primed CD56 $6^{\text {bright }} \mathrm{NK}$ cells were incubated with CFSE-labeled, TRAIL-sensitive (HL-60, RPMI 8226) or -insensitive (U266) targets for 18 hours $(79,80)$ at a 5:1 E:T ratio in the presence of $10 \mu \mathrm{g} / \mathrm{ml}$ antiTRAIL or control IgG antibody. Background spontaneous death was less than 5\% (U266, HL60) or 10\% (RPMI 8226).

Light microscopy. Slides were prepared from $1 \times 10^{5}$ to $2 \times 10^{5}$ cells (via CytoSpin) and Wright-Giemsa-stained. Micrographs were obtained using Nikon Microphot SA at $\times 1,000$, with no postimage alterations except cropping for image size. Scale bar represents $5 \mu \mathrm{m}$.

Confocal immunofluorescence microscopy. Flow-sorted CD56 bright NK cells were cultured for 16 hours in X-vivo20 media (Lonza), supplemented with $5 \%$ human serum with or without rhIL-15 at $5 \mathrm{ng} /$ $\mathrm{ml}$. The cells were adhered to CellTak-coated (Corning) coverslips, fixed with $4 \%$ paraformaldehyde, and stained for 4 hours or overnight with rabbit anti-human LAMP1 (Ab24170) and mouse anti-human granzyme B-A647 (GB11), followed by donkey anti-rabbit IgG Alexa Fluor 568 and Hoechst 33342. The stained cells were mounted with ProLong Diamond Antifade on coverslips. Z-stacks were acquired on an LSM710 (Zeiss) confocal microscope equipped with a laser diode 405-30 CW (405 nm), an Ar-Laser Multiline (458/488/514 nm), a DPSS-561 $10(561 \mathrm{~nm})$, and a He-Ne laser (633 nm). A Plan-Apochromat $63 \times$ NA/1.4 oil DICII (Zeiss) objective was used for the acquisition. All images were acquired using the same confocal settings. Image processing was conducted with Zen2012 software (Zeiss). Single-cell quantification of mean fluorescence intensity $\times$ area was performed with Fiji, using the analyze particles function for all particles larger 
than $0.001 \mu \mathrm{m}^{2}$. Therefore, binary images were created and local thresholding was applied using the Bernsen algorithm in Image J (15-pixel radius, default parameters) and Watershed segmentation.

Tumor cell conjugation assay. Tumor conjugate formation was assessed by coincubation of purified NK cells with CFSE-labeled K562 cells for 5,15 , or 30 minutes. The frequency of CFSE-positive CD56 $6^{\text {bright }}$ NK events was used to calculate tumor conjugate percentages (81).

Integrin (LFA-1) assays. To assess open-conformation LFA-1 expression or signaling (pERK), control or IL-15-primed purified NK cells were coincubated with $\mathrm{K} 562$ tumor targets $(\mathrm{E}: \mathrm{T}=5: 1)$, spun at $400 \mathrm{~g}$ for 1 minute, then placed at $37^{\circ} \mathrm{C}$ for 3 minutes (pERK) or $15 \mathrm{~min}$ utes (open LFA-1). To stain for pERK, cells were fixed in $1.7 \%$ paraformaldehyde, permeabilized in 100\% methanol, then stained overnight for NK cell surface markers and pERK1/2 (pT202, pY204). For openconformation LFA-1 expression, cells were surface-stained for NK cell markers and LFA-1 (clone m24, specific for the open, high-affinity conformation of LFA-1; ref. 82).

NSG xenograft model. CD56 ${ }^{\text {bright }}$ NK cells were flow-sorted from normal-donor purified NK cells on day -1 , then primed with $17.5 \mathrm{ng} / \mathrm{ml}$ ALT-803 (equivalent to $5 \mathrm{ng} / \mathrm{ml}$ rhIL-15) for 16 hours. Cells were harvested for i.v. injection ( $200 \mu \mathrm{l}$ in PBS via tail vein) into 8- to 12-week-old male and female NOD-SCID-IL2R $\gamma^{-1-}$ (NSG) mice (The Jackson Laboratory) on day 0. Eight mice received an equal number $\left(0.5 \times 10^{6}\right.$ to 0.66 $\times 10^{6}$ ) of K562-luciferase (K562-luc) tumor cells and ALT-803-primed CD56 $6^{\text {bright }} \mathrm{NK}$ cells, and 8 mice received the same amount of tumor without NK cells. In addition, $5 \mu \mathrm{g}$ of ALT- 803 was administered via tail vein at the time of adoptive cell transfer and every 2-3 days after for the first 30 days. Mice were imaged by bioluminescence imaging on days 2, $5,13,20$, and 27 ( \pm 1 day). Bioluminescence imaging was performed as previously described (47) with exposure times of 1-180 seconds.

IL-15 signaling pathway phosphorylation assays. Purified NK cells were incubated with $5 \mathrm{ng} / \mathrm{ml}$ IL-15 for 2 hours (pAkt and pERK) or 30 minutes (pSTAT5). Next, cells were fixed in $1.7 \%$ paraformaldehyde, permeabilized in $100 \%$ methanol, then stained overnight for NK cell surface markers and phospho-signaling molecules.

Flow cytometric analysis. Cell staining was performed as previously described (53), and data were acquired on a Gallios flow cytometer (Beckman Coulter) and analyzed using Kaluza (Beckman Coulter) or FlowJo (Tree Star) software.

Statistics. Statistical comparisons were performed using a paired Student's $t$ test, 1-way ANOVA, 1-way repeated-measures ANOVA, or 2-way ANOVA with post hoc analyses, where appropriate. A $P$ value less than 0.05 was considered significant $\left({ }^{*} P<0.05,{ }^{* *} P<0.01,{ }^{* * *} P<0.001\right)$.

Study approval. Patients with newly diagnosed AML provided informed consent under the Washington University IRB-approved protocol 2010-11766 for these studies (Supplemental Table 2). Five patients with newly diagnosed MM provided informed consent under the Washington University IRB-approved protocol 2011-02270 for these studies (Supplemental Table 3). For 1 patient, PBMCs were available only after 1 round of therapy (4 cycles of Revlimid/Velcade/dexamethasone [RVD] followed by melphalan-conditioned autologous stem cell transplantation). For the above patients, PBMCs or tumor cells were viably cryopreserved and thawed for use in assays.

Five patients with relapsed/refractory MM were treated on a phase I study of ALT-803 and provided informed consent for sample collection under IRB-approved protocol 2011-02270 (Supplemental Table 4). Two of the 5 patients were treated with $10 \mu \mathrm{g} / \mathrm{kg}$ ALT- 803 s.c. (patient group A), and 3 were treated with 3-6 $\mu$ g/ $\mathrm{kg}$ ALT-803 i.v. (patient group B). One patient from group A was sampled at 2 independent time points. Peripheral blood samples were drawn before ALT-803 and approximately 24 and 72 hours after the first dose of ALT-803, and functional assays were performed on freshly isolated PBMCs. All patients provided informed consent prior to sample collection and/or treatment.

NSG mice were maintained under specific pathogen-free conditions and used in accordance with our animal protocol approved by the Washington University Animal Studies Committee.

\section{Note added in proof}

While this paper was in press, data from a clinical trial further corroborated our findings by demonstrating that continuous rhIL-15 infusion enhances cytotoxic CD56 $6^{\text {bright }} \mathrm{NK}$ cells in vivo in patients with cancer (83).

\section{Author contributions}

JAW, RR, MR, and TAF conceived and designed the study. JAW, RR, MR, MMBE, SES, JWL, RPS, BAJ, MBH, TS, SAL, ARI, DJ, JAK, RV, DC, JG, KJM, and HCW collected, analyzed, and assembled the data or provided patient samples. JAW, RR, and TAF wrote the manuscript. All authors reviewed the data and edited and approved the final version of the manuscript.

\section{Acknowledgments}

This work was supported by grants from the Howard Hughes Medical Institute (HHMI; Medical Fellow Award, to JAW), an American Society of Hematology Scholar Award (to RR), an American Society of Clinical Oncology Career Development Award (to RR), $\mathrm{NIH} /$ National Cancer Institute (NCI) F32CA200253 (to MMBE), NIH T32HL007088 (to JAW and RPS), a Siteman Cancer Center SIP Award (to TAF), an HHMI Physician Scientist Early Career Award (to TAF), a Leukemia SPORE (P50CA171963) Developmental Research Award (to TAF), NIH R01AI102924 (to TAF), the Swedish Research Council (to KJM), the Swedish Children's Cancer Society (to KJM), the Swedish Cancer Center (to KJM), the Norwegian Research Council (to KJM), the Norwegian Cancer Society (to KJM), the South-Eastern Norway Regional Health Authority (to KJM), and Stiftelsen KG Jebsen (to KJM). The Siteman Cancer Center Flow Cytometry Core and Immunomonitoring Laboratory were used for this study, supported by NCI Cancer Center Support Grant P30CA91842, and the CHiiPs Center at Washington University. The authors thank Washington University (P01CA101937 and P50CA171963) for supporting access to primary AML patient samples. We thank the Core Facility of Advanced Light Microscopy and the Flow Cytometry Core Facility at the Oslo University Hospital Institute for Cancer Research. We thank Megan Cooper, Anthony French, Marco Colonna, and Wayne Yokoyama for insightful discussion.

Address correspondence to: Todd A. Fehniger, Division of Oncology, Department of Medicine, Washington University School of Medicine, 660 S. Euclid Avenue, Campus Box 8007, St. Louis, Missouri 63110, USA. Phone: 314.362.5654; Email: tfehnige@wustl.edu.

RPS's present address is: Novartis Institutes for Biomedical Research, Cambridge, Massachusetts, USA. 
1. Caligiuri MA. Human natural killer cells. Blood. 2008;112(3):461-469.

2. Lanier LL. NK cell recognition. Annu Rev Immunol. 2005;23:225-274.

3. Long EO, Kim HS, Liu D, Peterson ME, Rajagopalan S. Controlling natural killer cell responses: integration of signals for activation and inhibition. Annu Rev Immunol. 2013;31:227-258.

4. Min-Oo G, Kamimura Y, Hendricks DW, Nabekura T, Lanier LL. Natural killer cells: walking three paths down memory lane. Trends Immunol. 2013;34(6):251-258.

5. Vidal SM, Khakoo SI, Biron CA. Natural killer cell responses during viral infections: flexibility and conditioning of innate immunity by experience. Curr Opin Virol. 2011;1(6):497-512.

6. Jonsson AH, Yokoyama WM. Natural killer cell tolerance licensing and other mechanisms. $A d v$ Immunol. 2009;101:27-79.

7. Cooper MA, Fehniger TA, Caligiuri MA. The biology of human natural killer-cell subsets. Trends Immunol. 2001;22(11):633-640.

8. Freud AG, Caligiuri MA. Human natural killer cell development. Immunol Rev. 2006;214:56-72.

9. Fehniger TA, et al. CD56bright natural killer cells are present in human lymph nodes and are activated by T cell-derived IL-2: a potential new link between adaptive and innate immunity. Blood. 2003;101(8):3052-3057.

10. Ferlazzo G, et al. The abundant NK cells in human secondary lymphoid tissues require activation to express killer cell Ig-like receptors and become cytolytic. J Immunol. 2004;172(3):1455-1462.

11. Ferlazzo G, et al. Distinct roles of IL-12 and IL-15 in human natural killer cell activation by dendritic cells from secondary lymphoid organs. Proc Natl Acad Sci U S A. 2004;101(47):16606-16611.

12. Fehniger TA, et al. Differential cytokine and chemokine gene expression by human NK cells following activation with IL-18 or IL-15 in combination with IL-12: implications for the innate immune response. JImmunol. 1999;162(8):4511-4520.

13. Cooper MA, et al. Human natural killer cells: a unique innate immunoregulatory role for the CD56(bright) subset. Blood. 2001;97(10):3146-3151.

14. Ruggeri $\mathrm{L}$, et al. Effectiveness of donor natural killer cell alloreactivity in mismatched hematopoietic transplants. Science. 2002;295(5562):2097-2100.

15. Miller JS, et al. Successful adoptive transfer and in vivo expansion of human haploidentical NK cells in patients with cancer. Blood. 2005;105(8):3051-3057.

16. Rubnitz JE, et al. NKAML: a pilot study to determine the safety and feasibility of haploidentical natural killer cell transplantation in childhood acute myeloid leukemia. JClin Oncol. 2010;28(6):955-959.

17. Ljunggren HG, Malmberg KJ. Prospects for the use of NK cells in immunotherapy of human cancer. Nat Rev Immunol. 2007;7(5):329-339.

18. Berrien-Elliott MM, Romee R, Fehniger TA. Improving natural killer cell cancer immunotherapy. Curr Opin Organ Transplant. 2015;20(6):671-680.

19. Cooley S, et al. Donors with group B KIR haplotypes improve relapse-free survival after unrelated hema- topoietic cell transplantation for acute myelogenous leukemia. Blood.2009;113(3):726-732.

20. Venstrom JM, et al. HLA-C-dependent prevention of leukemia relapse by donor activating KIR2DS1. N Engl JMed. 2012;367(9):805-816.

21. Cooley S, et al. Donor selection for natural killer cell receptor genes leads to superior survival after unrelated transplantation for acute myelogenous leukemia. Blood. 2010;116(14):2411-2419.

22. Fehniger TA, Caligiuri MA. Interleukin 15: biology and relevance to human disease. Blood. 2001;97(1):14-32.

23. Budagian V, Bulanova E, Paus R, Bulfone-Paus S. IL-15/IL-15 receptor biology: a guided tour through an expanding universe. Cytokine Growth Factor Rev. 2006;17(4):259-280

24. Mishra A, Sullivan L, Caligiuri MA. Molecular pathways: interleukin-15 signaling in health and in cancer. Clin Cancer Res. 2014;20(8):2044-2050.

25. Fehniger TA, Cooper MA, Caligiuri MA. Interleukin-2 and interleukin-15: immunotherapy for cancer. Cytokine Growth Factor Rev. 2002;13(2):169-183.

26. Koreth J, et al. Interleukin-2 and regulatory $\mathrm{T}$ cells in graft-versus-host disease. $N$ Engl J Med. 2011;365(22):2055-2066.

27. Boyman O, Sprent J. The role of interleukin-2 during homeostasis and activation of the immune system. Nat Rev Immunol. 2012;12(3):180-190.

28. Fehniger TA, et al. Acquisition of murine NK cell cytotoxicity requires the translation of a pre-existing pool of granzyme $B$ and perforin mRNAs. Immunity. 2007;26(6):798-811.

29. Lucas M, Schachterle W, Oberle K, Aichele P, Diefenbach A. Dendritic cells prime natural killer cells by trans-presenting interleukin 15 . Immunity. 2007;26(4):503-517.

30. Nielsen CM, White MJ, Goodier MR, Riley EM. Functional significance of CD57 expression on human NK cells and relevance to disease. Front Immunol. 2013;4:422.

31. Huntington ND, Vosshenrich CA, Di Santo JP. Developmental pathways that generate naturalkiller-cell diversity in mice and humans. Nat Rev Immunol. 2007;7(9):703-714.

32. Amir el-AD, et al. viSNE enables visualization of high dimensional single-cell data and reveals phenotypic heterogeneity of leukemia. Nat Biotechnol. 2013;31(6):545-552.

33. Orange JS. Formation and function of the lytic NK-cell immunological synapse. Nat Rev Immunol. 2008;8(9):713-725.

34. Jacobs R, et al. CD56bright cells differ in their KIR repertoire and cytotoxic features from CD $56^{\mathrm{dim}} \mathrm{NK}$ cells. Eur J Immunol. 2001;31(10):3121-3127.

35. Lincz LF, Yeh TX, Spencer A. TRAIL-induced eradication of primary tumour cells from MM patient bone marrows is not related to TRAIL receptor expression or prior chemotherapy. Leukemia. 2001;15(10):1650-1657.

36. Plasilova M, et al. TRAIL (Apo2L) suppresses growth of primary human leukemia and myelodysplasia progenitors. Leukemia. 2002;16(1):67-73.

37. Anguille S, et al. Interleukin-15 dendritic cells harness NK cell cytotoxic effector function in a contact- and IL-15-dependent manner. PLoS One. 2015;10(5):e0123340
38. Keating SE, et al. Metabolic reprogramming supports IFN- $\gamma$ production by $\mathrm{CD} 56^{\text {bright }} \mathrm{NK}$ cells. Jimmunol. 2016;196(6):2552-2560.

39. Mace EM, et al. Cell biological steps and checkpoints in accessing NK cell cytotoxicity. Immunol Cell Biol. 2014;92(3):245-255.

40. Barber DF, Faure M, Long EO. LFA-1 contributes an early signal for NK cell cytotoxicity. J Immunol. 2004;173(6):3653-3659.

41. Abram CL, Lowell CA. The ins and outs of leukocyte integrin signaling. Annu Rev Immunol. 2009;27:339-362.

42. Urlaub D, Höfer K, Müller ML, Watzl C. LFA-1 activation in NK cells and their subsets: influence of receptors, maturation, and cytokine stimulation. J Immunol. 2017;198(5):1944-1951.

43. Perez OD, Mitchell D, Jager GC, Nolan GP. LFA-1 signaling through $\mathrm{p} 44 / 42$ is coupled to perforin degranulation in $\mathrm{CD} 56^{+} \mathrm{CD} 8^{+}$natural killer cells. Blood. 2004;104(4):1083-1093.

44. Waldmann TA. Interleukin-15 in the treatment of cancer. Expert Rev Clin Immunol. 2014;10(12):1689-1701.

45. Conlon KC, et al. Redistribution, hyperproliferation, activation of natural killer cells and CD8 $\mathrm{T}$ cells, and cytokine production during first-inhuman clinical trial of recombinant human interleukin-15 in patients with cancer. JClin Oncol. 2015;33(1):74-82.

46. Rhode PR, et al. Comparison of the superagonist complex, ALT-803, to IL15 as cancer immunotherapeutics in animal models. Cancer Immunol Res. 2016;4(1):49-60.

47. Rosario M, et al. The IL-15-based ALT-803 complex enhances Fc $\gamma$ RIIIa-triggered NK cell responses and in vivo clearance of B cell lymphomas. Clin Cancer Res. 2016;22(3):596-608.

48. Zhu X, et al. Novel human interleukin-15 agonists. JImmunol. 2009;183(6):3598-3607.

49. Nandagopal N, Ali AK, Komal AK, Lee SH. The critical role of IL-15-PI3K-mTOR pathway in natural killer cell effector functions. Front Immunol. 2014;5:187.

50. Marçais A, et al. The metabolic checkpoint kinase mTOR is essential for IL-15 signaling during the development and activation of NK cells. Nat Immunol. 2014;15(8):749-757.

51. Reynolds AM. The Cahn-Hilliard phase separation principle maybe the tip of an iceberg: comment on "Phase separation driven by densitydependent movement: a novel mechanism for ecological patterns" by Q.-X. Liu et al. Phys Life Rev. 2016;19:135-136

52. Romee R, Leong JW, Fehniger TA. Utilizing cytokines to function-enable human NK cells for the immunotherapy of cancer. Scientifica (Cairo). 2014;2014:205796.

53. Romee R, et al. Cytokine activation induces human memory-like NK cells. Blood. 2012;120(24):4751-4760

54. Cooper MA, Elliott JM, Keyel PA, Yang L, Carrero JA, Yokoyama WM. Cytokine-induced memorylike natural killer cells. Proc Natl Acad Sci U S A. 2009;106(6):1915-1919.

55. Ni J, Miller M, Stojanovic A, Garbi N, Cerwenka A. Sustained effector function of IL-12/15/18preactivated NK cells against established tumors. JExp Med. 2012;209(13):2351-2365. 
56. Romee R, et al. Cytokine-induced memory-like natural killer cells exhibit enhanced responses against myeloid leukemia. Sci Transl Med. 2016;8(357):357ra123.

57. Benjamin JE, Gill S, Negrin RS. Biology and clinical effects of natural killer cells in allogeneic transplantation. Curr Opin Oncol. 2010;22(2):130-137.

58. Messaoudene M, et al. Mature cytotoxic CD56(bright)/CD16(+) natural killer cells can infiltrate lymph nodes adjacent to metastatic melanoma. Cancer Res. 2014;74(1):81-92.

59. Strowig T, et al. Tonsilar NK cells restrict B cell transformation by the Epstein-Barr virus via IFN- $\gamma$. PLoS Pathog. 2008;4(2):e27.

60. Briard D, Brouty-Boyé D, Azzarone B, Jasmin C. Fibroblasts from human spleen regulate NK cell differentiation from blood CD34(+) progenitors via cell surface IL-15. J Immunol. 2002;168(9):4326-4332.

61. Soderquest K, et al. Monocytes control natural killer cell differentiation to effector phenotypes. Blood. 2011;117(17):4511-4518.

62. Robertson MJ, et al. Response of human natural killer (NK) cells to NK cell stimulatory factor (NKSF): cytolytic activity and proliferation of NK cells are differentially regulated by NKSF. J Exp Med.1992;175(3):779-788.

63. Takahashi E, et al. Induction of $\mathrm{CD} 16^{+} \mathrm{CD} 56^{\text {bright }}$ NK cells with antitumour cytotoxicity not only from $\mathrm{CD}^{-} 6^{-} \mathrm{CD} 56^{\text {bright }} \mathrm{NK}$ cells but also from CD16- CD56 ${ }^{\mathrm{dim}}$ NK cells. Scand J Immunol. 2007;65(2):126-138.

64. Nielsen N, Ødum N, Ursø B, Lanier LL, Spee P. Cytotoxicity of CD56 (bright) NK cells towards autologous activated $\mathrm{CD} 4^{+} \mathrm{T}$ cells is mediated through NKG2D, LFA-1 and TRAIL and dampened via CD94/NKG2A. PLoS One. 2012;7(2):e31959.

65. Mao Y, et al. IL-15 activates mTOR and primes stress-activated gene expression leading to prolonged antitumor capacity of NK cells. Blood. 2016;128(11):1475-1489.

66. Trotta R, et al. Differential expression of SHIP1 in CD56bright and CD56dim NK cells provides a molecular basis for distinct functional responses to monokine costimulation. Blood. 2005;105(8):3011-3018.

67. Briercheck EL, et al. PTEN is a negative regulator of NK cell cytolytic function. J Immunol. 2015;194(4):1832-1840.

68. Grzywacz B, Miller JS, Verneris MR. Use of natural killer cells as immunotherapy for leukaemia. Best Pract Res Clin Haematol. 2008;21(3):467-483.

69. Zhang H, et al. Activating signals dominate inhibitory signals in CD137L/IL-15 activated natural killer cells. JImmunother. 2011;34(2):187-195.

70. Cooley S, et al. Recombinant human IL-15 promotes in vivo expansion of adoptively transferred NK cells in a first-in-human phase I dose escalation study in patients with AML. Blood. 2012;120(21):A894.

71. Rosario M, et al. The IL-15-based ALT-803 complex enhances Fc $\gamma$ RIIIa-triggered NK cell responses and in vivo clearance of B cell lymphomas. Clin Cancer Res. 2016;22(3):596-608.

72. Rhode PR, et al. Comparison of the superagonist complex, ALT-803, to IL15 as cancer immunotherapeutics in animal models. Cancer Immunol Res. 2016;4(1):49-60.

73. Nguyen S, et al. NK-cell reconstitution after haploidentical hematopoietic stem-cell transplantations: immaturity of NK cells and inhibmitory effect of NKG2A override GvL effect. Blood. 2005;105(10):4135-4142.

74. Yu J, et al. Breaking tolerance to self, circulating natural killer cells expressing inhibitory KIR for non-self HLA exhibit effector function after T cell-depleted allogeneic hematopoietic cell transplantation. Blood. 2009;113(16):3875-3884.
75. Björklund AT, et al. NK cells expressing inhibitory KIR for non-self-ligands remain tolerant in HLAmatched sibling stem cell transplantation. Blood. 2010;115(13):2686-2694.

76. Vukicevic M, et al. CD56 $6^{\text {bright }}$ NK cells after hematopoietic stem cell transplantation are activated mature NK cells that expand in patients with low numbers of T cells. Eur J Immunol. 2010;40(11):3246-3254.

77. Foley B, et al. Cytomegalovirus reactivation after allogeneic transplantation promotes a lasting increase in educated NKG2C+ natural killer cells with potent function. Blood. 2012;119(11):2665-2674.

78. Dulphy N, et al. An unusual CD56(bright) CD16(low) NK cell subset dominates the early posttransplant period following HLA-matched hematopoietic stem cell transplantation. J Immunol. 2008;181(3):2227-2237.

79. Sarhan D, et al. Activated monocytes augment TRAIL-mediated cytotoxicity by human NK cells through release of IFN- $\gamma$. Eur J Immunol. 2013;43(1):249-257.

80. Wennerberg E, et al. Doxorubicin sensitizes human tumor cells to NK cell- and T-cell-mediated killing by augmented TRAIL receptor signaling. Int J Cancer. 2013;133(7):1643-1652.

81. Zheng X, Wang Y, Wei H, Sun R, Tian Z. LFA-1 and $\mathrm{CD} 2$ synergize for the Erk1/2 activation in the natural killer (NK) cell immunological synapse. J Biol Chem. 2009;284(32):21280-21287.

82. Dransfield I, Hogg N. Regulated expression of $\mathrm{Mg}^{2+}$ binding epitope on leukocyte integrin $\alpha$ subunits. EMBO J. 1989;8(12):3759-3765.

83. Dubois S, et al. IL15 infusion of cancer patients expands the subpopulation of cytotoxic CD $56^{\text {bright }}$ NK cells and increases NK cell cytokine release capabilities [published online ahead of print August 25, 2017]. Cancer Immunol Res. https:// doi.org/10.1158/2326-6066.CIR-17-0279. 